\title{
The extreme chemistry of multiple stellar populations in the metal-poor globular cluster NGC $4833^{\star, \star \star}$
}

\author{
E. Carretta ${ }^{1}$, A. Bragaglia ${ }^{1}$, R. G. Gratton ${ }^{2}$, V. D’Orazi ${ }^{3,4}$, S. Lucatello ${ }^{2}$, Y. Momany ${ }^{2,5}$, A. Sollima ${ }^{1}$, M. Bellazzini ${ }^{1}$, \\ G. Catanzaro ${ }^{6}$, and F. Leone ${ }^{7}$
}

\author{
1 INAF - Osservatorio Astronomico di Bologna, via Ranzani 1, 40127 Bologna, Italy \\ e-mail: eugenio.carretta@oabo.inaf.it \\ 2 INAF - Osservatorio Astronomico di Padova, Vicolo dell'Osservatorio 5, 35122 Padova, Italy \\ 3 Dept. of Physics and Astronomy, Macquarie University, NSW 2109 Sydney, Australia \\ ${ }^{4}$ Monash Centre for Astrophysics, Monash University, School of Mathematical Sciences, Building 28, VIC 3800 Clayton, \\ Melbourne, Australia \\ 5 European Southern Observatory, Alonso de Cordova 3107, Casilla 19001, Vitacura, Santiago, Chile \\ ${ }^{6}$ INAF-Osservatorio Astrofisico di Catania, via S. Sofia 78, 95123 Catania, Italy \\ 7 Dipartimento di Fisica e Astronomia, Università di Catania, via S. Sofia 78, 95123 Catania, Italy
}

Received 23 December 2013 / Accepted 27 January 2014

\begin{abstract}
Our FLAMES survey of Na-O anticorrelation in globular clusters (GCs) is extended to NGC 4833, a metal-poor GC with a long blue tail on the horizontal branch (HB). We present the abundance analysis for a large sample of 78 red giants based on UVES and GIRAFFE spectra acquired at the ESO-VLT. We derived abundances of $\mathrm{Na}, \mathrm{O}, \mathrm{Mg}, \mathrm{Al}, \mathrm{Si}, \mathrm{Ca}, \mathrm{Sc}, \mathrm{Ti}, \mathrm{V}, \mathrm{Cr}, \mathrm{Mn}, \mathrm{Fe}, \mathrm{Co}, \mathrm{Ni}, \mathrm{Cu}, \mathrm{Zn}$, $\mathrm{Y}, \mathrm{Ba}, \mathrm{La}$, and Nd. This is the first extensive study of this cluster from high resolution spectroscopy. On the scale of our survey, the metallicity of NGC 4833 is $[\mathrm{Fe} / \mathrm{H}]=-2.015 \pm 0.004 \pm 0.084 \mathrm{dex}(\mathrm{rms}=0.014 \mathrm{dex})$ from 12 stars observed with UVES, where the first error is from statistics and the second one refers to the systematic effects. The iron abundance in NGC 4833 is homogeneous at better than $6 \%$. On the other hand, the light elements involved in proton-capture reactions at high temperature show the large star-to-star variations observed in almost all GCs studied so far. The Na-O anticorrelation in NGC 4833 is quite extended, as expected from the high temperatures reached by stars on the $\mathrm{HB}$, and NGC 4833 contains a conspicuous fraction of stars with extreme [O/Na] ratios. More striking is the finding that large star-to-star variations are also seen for $\mathrm{Mg}$, which spans a range of more than 0.5 dex in this GC. Depletions in $\mathrm{Mg}$ are correlated to the abundances of $\mathrm{O}$ and anti-correlated with $\mathrm{Na}, \mathrm{Al}$, and $\mathrm{Si}$ abundances. This pattern suggests the action of nuclear processing at unusually high temperatures, producing the extreme chemistry observed in the stellar generations of NGC 4833. These extreme changes are also seen in giants of the much more massive GCs M 54 and $\omega$ Cen, and our conclusion is that NGC 4833 has probably lost a conspicuous fraction of its original mass due to bulge shocking, as also indicated by its orbit.
\end{abstract}

Key words. stars: abundances - stars: atmospheres - stars: Population II - globular clusters: general globular clusters: individual: NGC 4833

\section{Introduction}

Globular clusters (GCs) are the brightest relics of the early phases of galaxy formation. Their study provides basic information on the early evolution of their host galaxy. In the past few years, it has become apparent that the episodes leading to the formation of these massive objects were complex. The main evidence for this complexity comes from the presence of chemical inhomogeneities that in most cases are limited to light elements involved in H-burning at high temperature ( $\mathrm{He}, \mathrm{CNO}, \mathrm{Na}$, $\mathrm{Mg}, \mathrm{Al}$ ), though in a few other GCs, star-to-star variations of heavier elements are also found (see reviews by Gratton et al. 2004, 2012). The extensive spectroscopic survey we are conducting (see e.g. Carretta et al. 2009a,b) revealed that these inhomogeneities are ubiquitous among GCs, but their range changes from cluster to cluster, being driven mainly by the total mass of

\footnotetext{
* Based on observations collected at ESO telescopes under programmes 083.D-0208 and 68.D-0265.

$\star \star$ Full Tables 2, 6-11 are only available at the CDS via anonymous ftp to cdsarc.u-strasbg. fr $(130.79 .128 .5)$ or via http://cdsarc.u-strasbg.fr/viz-bin/qcat?]/A+A/564/A60
}

the GCs, though metallicity and possibly other parameters also play a role.

Similar results have been obtained by other authors, using both similar analysis methods (e.g. Ramirez \& Cohen 2002, 2003; Marino et al. 2008; Johnson \& Pilachowski 2012) and photometric ones (e.g. Milone et al. 2012, 2013, and references therein). This has led to a general scenario where GCs host multiple stellar populations, often considered to correspond to different generations, where the younger stars (second-generation stars) are formed mainly or even exclusively from the ejecta of older ones (first or primordial generation stars: see e.g. D'Ercole et al. 2008; Decressin et al. 2008), though alternatives are also being considered (see e.g. Bastian et al. 2013a).

There are several key aspects that still require understanding. It is important to establish which of the first-generation stars polluted the material from which second-generation stars formed. This is relevant to setting both the involved time scale and the mass budget. Given that no variation in $\mathrm{Fe}$ abundance is observed in most GCs (see e.g. Carretta et al. 2009c), supernovae should play at most a very minor role, apart from a few exceptional cases. This clearly makes the chemical evolution of 
GCs very different from the one typically observed in galaxies, and requires a restriction of the mass range of the polluters. Several candidates have been proposed: the most massive among intermediate-mass stars during the asymptotic giant branch (AGB) phase (Ventura et al. 2001); fast-rotating massive stars (Decressin et al. 2007); massive binaries (de Mink et al. 2009); novae (Maccarone \& Zurek 2012).

Arguments for or against each of these candidates exist, and it is not even clear if they should be considered as mutually exclusive. Scenarios based on different polluters produce different expectations about some observables (e.g. Valcarce and Catelan 2011). For instance, those involving massive stars are characterized by a very short time scale, which is possibly supported by the lack of evidence for multiple generation of stars in presentday massive clusters (Bastian et al. 2013b), though it is not very clear that such objects are as massive as the Milky Way GCs were when they formed. On the other hand, they have difficulty producing a well-defined threshold in cluster mass for the phenomenon (see Carretta et al. 2010a), as well as clearly separated stellar populations, as observed in several typical clusters from both photometry and spectroscopy (NGC 2808: D'Antona et al. 2005; Carretta et al. 2006, 2009a,b, 2012a,b; Piotto et al. 2007; M 4: Marino et al. 2008; NGC 6752: Milone et al. 2013; 47 Tuc: Milone et al. 2012, to quote a few examples).

Providing high quality data on more GCs is clearly needed to strengthen the results obtained so far. Spectroscopic analysis of rather large samples of stars may, for instance, be used to show whether stars can be divided into discrete groups in chemical composition, which may be explained more easily as evidence of multiple episodes of star formation, or rather distribute continuously. Also, very stringent limits on star-to-star variations in $\mathrm{Fe}$ abundances as observed in many GCs by Carretta et al. (2009c) or Yong et al. (2013) severely limit the possibility that SNe contributed to chemical evolution, which is one of the basic problems to be faced by short-timescale scenarios of cluster formation.

Following our earlier work (e.g. Carretta et al. 2006, 2009a,b), we present here the results of the analysis of large samples of spectra in NGC 4833, focussing on the Na-O anticorrelation, but also providing data for $\mathrm{Fe}, \mathrm{Mg}, \mathrm{Al}, \mathrm{Si}, \mathrm{Ca}, \mathrm{Ti}$, and other elements. This is the first extensive analysis of high dispersion spectra for this cluster since only two red giant branch (RGB) stars were analysed by Gratton and Ortolani (1989) and one by Minniti et al. (1996), which of course prevented any discussion on chemical inhomogeneities. NGC $4833\left(M_{V}=-8.16\right.$ : Harris 1996) has been classified as an old halo cluster with a moderately extended blue horizontal branch (Mackey \& van den Bergh 2005) or an inner halo cluster (Carretta et al. 2010a). Studies of variable stars have been presented by Murphy \& Darragh (2012, 2013), who found 17 RR Lyrae and six SX Phoenicis stars. The mean period of the RR Lyrae allows classifying the cluster as Oosterhoff II. NGC 4833 is seen projected close to the Coal Sack, and has a moderately large reddening $(E(B-$ $V)=0.32$ ) with non-negligible variations across the cluster. Casetti-Dinescu et al. (2007) determined a very eccentric orbit $(e=0.84)$ that brings the cluster very close to the Galactic center and never far from the Galactic plane $\left(z_{\max }=1.8 \mathrm{kpc}\right.$, $\left.r_{a}=7.7 \mathrm{kpc}, r_{\mathrm{p}}=0.7 \mathrm{kpc}\right)$. Orbital parameters are similar to those of NGC 5986, so that a tentative association between the two clusters was proposed by Casetti-Dinescu et al.

The structure of this paper is as follows. Observations, radial velocities, and kinematics are presented in Sect. 2, while Sect. 3 is devoted to the abundance analysis, whose results are illustrated in Sect. 4. Our findings are discussed in Sect. 5 and summarised in Sect. 6.

\section{Observations}

The photometric catalogue for NGC 4833 is based on $U B V I$ data (ESO programme 68.D-0265, PI Ortolani), collected at the Wide-Field Imager at the 2.2-m ESO-MPI telescope on 17-21 February 2002. The WFI covers a total field of view of $34^{\prime} \times 33^{\prime}$, consisting of $8,2048 \times 4096$ EEV-CCDs with a pixel size of 0.238 . The collected images were dithered to cover the gaps between the CCDs, and the exposure times were divided into shallow and deep so as not to saturate the bright red giant stars and, at the same time, sample the faint main sequence stars with a good signal-to-noise ratio $(\mathrm{S} / \mathrm{N})$.

The de-biasing and flat-fielding reduction of the CCD mosaic raw images employed the IRAF package MSCRED (Valdes 1998), while the stellar photometry was derived by using the DAOPHOT and ALLFRAME programs (Stetson 1994). For specific details of the photometric reduction process see Momany et al. (2004).

The absolute photometric calibration primarily employed the use of $U B V I$ standard stars from Landolt (1992), as well as secondary stars from the Stetson library ${ }^{1}$, which provides more numerous and relatively fainter $B V I$ standards. The uncertainties in the absolute flux calibration were of the order of $0.06,0.03,0.03$, and $0.04 \mathrm{mag}$ for $U B V I$, respectively. The NGC 4833 catalogue was not corrected for sky concentration, caused by the spurious reflections of light and its subsequent redistribution in the focal plane (Manfroid et al. 2001). As a consequence, photometric comparisons to independent catalogues (based on a smaller field of view data) might reveal systematic offsets as a function of the distance from the centre of the WFI mosaic.

We selected a pool of stars lying near the RGB ridge line in the colour-magnitude diagram (CMD) and without close neighbours, i.e. without any star closer than 3 arcsec. (We also included cases with neighbours between 2 and 3 arcsec, but only if fainter by more than 2 mag.) The FPOss tool was used to allocate the FLAMES (Pasquini et al. 2002) fibres.

The stars in our spectroscopic sample are indicated in Fig. 1. We clearly see that NGC 4833 is heavily contaminated by field stars of the Galactic disk and bulge, given its present location at $l=303.60 \mathrm{deg}, b=-8.02 \mathrm{deg}$. Even if the reddening toward the cluster is high and differential (Melbourne \& Guhathakurta 2004), thus complicating the analysis, the excision of non-members via radial velocity and the use of infrared filters for temperature determinations, as done in similar cases (see e.g. Gratton et al. 2006, 2007), make it feasible.

The log of the observations is given in Table 1. We obtained two exposures with the high resolution GIRAFFE grating and the setup HR1 1 covering the Na I 5682-88 $\AA$ doublet and two exposures with the setup HR13, including the [O I] forbidden lines at 6300-63 A. Excluding a non-member star and another that was not useful owing to the low $\mathrm{S} / \mathrm{N}$ of the spectrum, we observed a total of 12 (bright) giants with the fibres feeding the UVES spectrograph (Red Arm, with spectral range from 4800 to $6800 \AA$ and $R=47000$, indicated as blue squares in Fig. 1) and 73 with GIRAFFE (seven are in common). The median values of the S/N of spectra obtained with UVES and with the HR11 and HR13 setups of GIRAFFE are 90, 95, and 140, respectively.

We used the $1 \mathrm{D}$, wavelength calibrated spectra as reduced by the ESO personnel with the dedicated FLAMES pipelines.

http://cadcwww.hia.nrc.ca/standards/ 


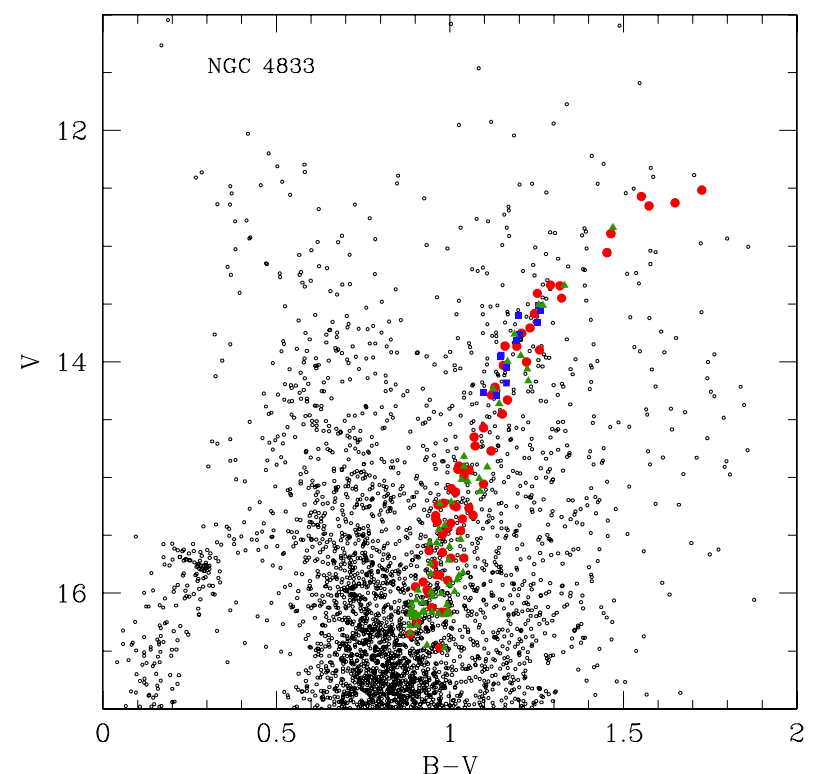

Fig. 1. $V, B-V C M D$ of NGC 4833 (open circles). Stars selected for the present study are plotted as filled, larger symbols: blue squares are the stars observed with UVES, red circles are stars with GIRAFFE spectra, and green triangles are stars observed with GIRAFFE but not analysed because found to be non-members from their RV (see text).

Table 1. Log of FLAMES observations for NGC 4833.

\begin{tabular}{lccccc}
\hline \hline Date & UT & $\begin{array}{c}\text { Exp. } \\
(\mathrm{s})\end{array}$ & $\begin{array}{c}\text { Grating } \\
\text { (") }\end{array}$ & $\begin{array}{c}\text { Seeing } \\
\text { Airmass }\end{array}$ \\
\hline Apr. 05, 2009 & $02: 11: 37.395$ & 2700 & HR11 & 1.07 & 1.599 \\
Apr. 05, 2009 & $02: 58: 02.303$ & 2700 & HR11 & 1.09 & 1.519 \\
Apr. 05, 2009 & $03: 54: 25.968$ & 2700 & HR13 & 0.64 & 1.462 \\
Apr. 05, 2009 & $04: 40: 47.286$ & 2700 & HR13 & 0.59 & 1.446 \\
\hline
\end{tabular}

Radial velocities (RV) for stars observed with the GIRAFFE spectrograph were obtained using the IRAF ${ }^{2}$ task FXCORR, with appropriate templates, while those of the stars observed with UVES were derived with the IRAF task RVIDLINES.

The large RV of NGC 4833 makes it easy to isolate cluster stars from field interlopers. In Fig. 2 we show the histogram of the heliocentric RVs derived for all stars observed; the cluster is easily spotted as a narrow and isolated peak around $V_{r} \simeq 200 \mathrm{~km} \mathrm{~s}^{-1}$. We select the 78 stars with $180.0 \leq R V \leq$ $220 \mathrm{~km} \mathrm{~s}^{-1}$ as candidate cluster members. Their membership is fully confirmed by the following chemical analysis, since they all have the same iron abundance, within the uncertainties. The nearest non-member in the velocity space has $R V=$ $141.6 \pm 0.7 \mathrm{~km} \mathrm{~s}^{-1}, \sim 60 \mathrm{~km} \mathrm{~s}^{-1}$ apart from the systemic velocity of the cluster. A brief overview of the cluster kinematics is provided in Sect. 2.1.

Our optical $B, V$ photometric data were integrated with $K$-band magnitudes from the Point Source Catalogue of 2MASS (Skrutskie et al. 2006) to derive atmospheric parameters as described below, in Sect. 3.

Coordinates, magnitudes, and heliocentric RVs are shown in Table 2 (the full table is only available at CDS).

\footnotetext{
2 IRAF is distributed by the National Optical Astronomical Observatory, which is operated by the Association of Universities for Research in Astronomy, under contract with the National Science Foundation.
}

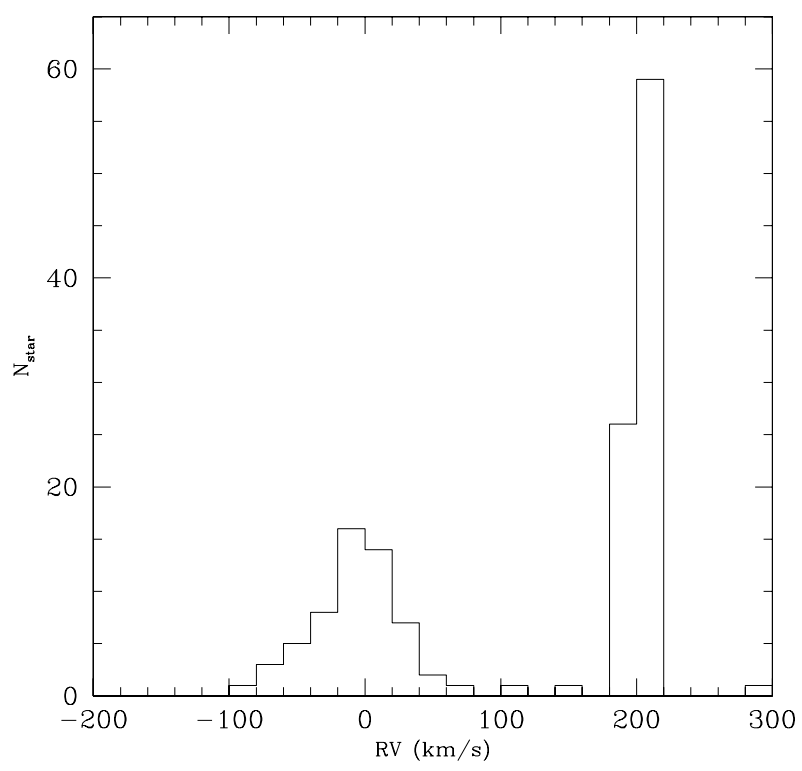

Fig. 2. Distribution of heliocentric radial velocities (RVs) for stars observed in NGC 4833.

\subsection{Radial velocities and kinematics}

There is no analysis in the literature dealing with the internal kinematics of NGC 4833, and no estimate of the central velocity dispersion $\sigma_{0}$. It is worth using our sample for a first investigation, although it is not especially well suited for this purpose. Indeed our 78 genuine cluster member stars are distributed (see Fig. 3) in the radial range $1.0 \leq R / r_{\mathrm{c}} \leq 6.6$ or $0.4 \leq R / r_{\mathrm{h}} \leq 2.8^{3}$. We therefore cannot sample the kinematics in the cluster core and in the outer halo. (The tidal radius is $r_{\mathrm{t}}=17.8 r_{\mathrm{c}}=7.4 r_{\mathrm{h}}$, since $c=1.25$.) Our analysis is fully homogenous with the one in Bellazzini et al. (2012) for the other clusters included in our survey: for further details, we address the interested reader to that paper. The only difference is that here mean velocity and velocity dispersions are estimated with the maximum likelihood (ML) procedure described in Martin et al. (2007), which naturally takes the effect of errors on the individual RV measures into account.

First of all, we compared the RV estimates obtained from HR13 and HR11 spectra for the 45 stars that have valid RV measures from both setups. The mean difference for the 35 stars brighter than $V=15.5$ (i.e. those with the smallest individual errors) is $\left\langle R V_{13}-R V_{11}\right\rangle=-0.43 \pm 0.07 \mathrm{~km} \mathrm{~s}^{-1}$. This shift was applied to the RV derived from HR11 spectra to bring all the RV estimates to a common zero point. Then, for each star, we adopted the RV estimated from HR13 spectra when present and when the individual uncertainty is lower than the HR11 estimate, and the RV from HR11 in the other cases.

Considering the whole sample of 78 stars, we find an average velocity of $\langle R V\rangle=202.0 \pm 0.5 \mathrm{~km} \mathrm{~s}^{-1}$ (and a dispersion of $4.1 \pm 0.3 \mathrm{~km} \mathrm{~s}^{-1}$ ), in very good agreement with the value reported by Harris (1996), $\langle R V\rangle=200 \pm 1.2 \mathrm{~km} \mathrm{~s}^{-1}$. Fitting a King (1966) model with $c=1.25$ to the velocity dispersion curve, we estimated the central velocity dispersion $\sigma_{0}=5.0 \pm 1.0 \mathrm{~km} \mathrm{~s}^{-1}$. We do not find any statistically significant rotation: in the notation of Bellazzini et al. (2012), the rotation amplitude is $A_{\text {rot }}=0.9 \pm 0.5 \mathrm{~km} \mathrm{~s}^{-1}$ and $A_{\text {rot }} / \sigma_{0}=0.18 \pm 0.11$.

3 The values for the core radius $r_{\mathrm{c}}$ and the half-light radius $r_{\mathrm{h}}$ were taken, as all the cluster parameters that will be used in the following, from the 2010 version of the Harris (1996) catalogue. 
Table 2. List and relevant information for target stars in NGC 4833.

\begin{tabular}{lccccccc}
\hline \hline ID & RA & Dec & $B$ & $V$ & $K$ & RV(Hel) & Notes \\
\hline 22810 & 12595.559 & -705659.87 & 16.470 & 15.491 & 12.337 & 198.79 & HR11, HR13 \\
23306 & 125849.698 & -705517.19 & 16.736 & 15.696 & 12.439 & 205.33 & HR11, HR13 \\
23437 & 125854.862 & -705459.07 & 16.153 & 15.056 & 11.711 & 195.26 & HR11 \\
23491 & 12595.197 & -705453.86 & 14.242 & 12.516 & 8.270 & 200.33 & HR11, HR13 \\
23518 & 125912.686 & -705450.03 & 16.395 & 15.359 & 12.206 & 203.73 & HR13 \\
24063 & 125854.127 & -705346.86 & 14.660 & 13.343 & 9.651 & 203.13 & HR13 \\
24252 & 125916.083 & -705328.91 & 14.916 & 13.663 & 10.046 & 202.26 & UVES \\
\hline
\end{tabular}

Notes. The complete table is available at the CDS.

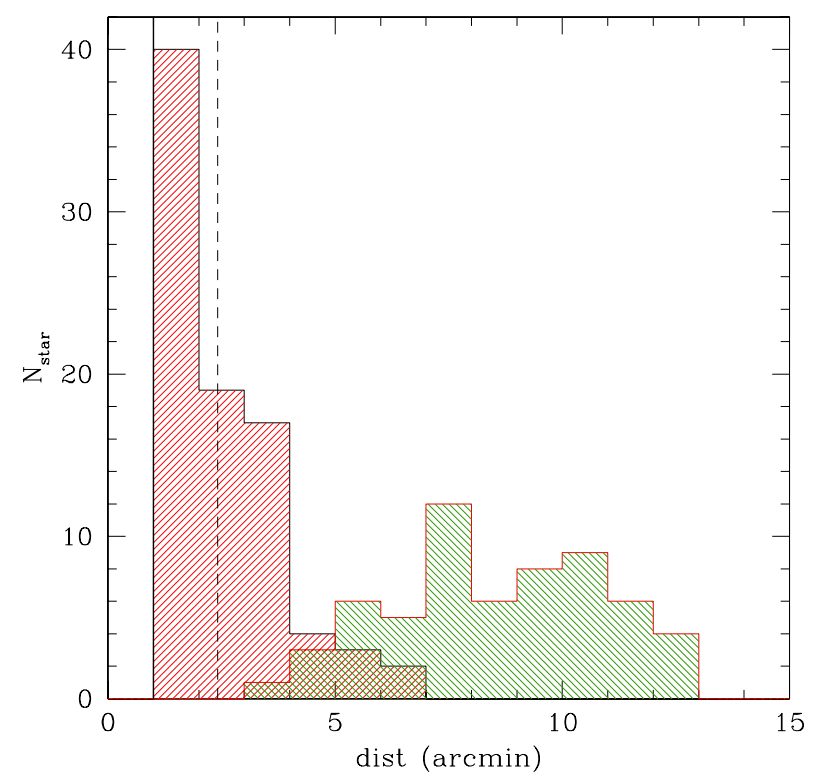

Fig. 3. Distribution of observed stars as a function of the radial distance from the centre of NGC 4833. Member stars are indicated in red, while green is used for non-members. The solid heavy line indicates the core radius $\left(r_{\mathrm{c}}=1 \mathrm{arcmin}\right)$, and the dashed line the half-mass radius $\left(r_{\mathrm{h}}=2.41 \mathrm{arcmin}\right)$ from Harris (1996).

As a result, NGC 4833 fits excellently into the correlations between metallicity (and horizontal branch morphology) and rotation found by Bellazzini et al.; i.e., metal-poor/blue HB clusters tend to have lower rotation than metal-rich/ red HB clusters.

Using the King (1966) formula, we estimate a dynamical mass $M_{\text {dyn }}=1.3_{-0.5}^{+0.6} \times 10^{5} M_{\odot}$, and $(M / L)_{V}=0.84 \pm 0.45$, on the low side of the range covered by Galactic GCs (see Sollima et al. 2012; Pryor \& Meylan 1993).

\section{Abundance analysis}

\subsection{Atmospheric parameters}

Following our tested procedure, effective temperatures $T_{\text {eff }}$ for our targets were derived using an average relation between apparent magnitudes and first-pass temperatures from $V-K$ colours and the calibrations of Alonso et al. (1999, 2001). The rationale behind this adopted procedure is to decrease the starto-star errors in abundances due to uncertainties in temperatures, since magnitudes are less affected by measure uncertainties than colours. In the case of NGC 4833, which is affected by high and variable reddening, we used the apparent $K$ magnitudes in our relation with $T_{\text {eff }}$ because the impact of the differential reddening on these magnitudes is very limited. This procedure worked very well on other GCs heavily affected by high and differential reddening patterns, such as the bulge clusters NGC 6441 (Gratton et al. 2006, 2007) and NGC 6388 (Carretta et al. 2007a). The adopted reddening $E(B-V)=0.32 \mathrm{mag}$ is from the Harris (1996) catalogue, and it is in perfect agreement with the value derived in the accurate study by Melbourne et al. (2000), who provided an associated error of $0.03 \mathrm{mag}$. Using Table 3 of Cardelli et al. (1989), this translates into an error of $0.01 \mathrm{mag}$ in $K$ magnitudes that, in turn, corresponds to an uncertainty in temperature of $2.14 \mathrm{~K}$, when coupled to the relation between effective temperature and $K$ magnitude adopted for NGC 4833 in the present study.

Gravities were obtained from apparent magnitudes, assuming the $T_{\text {eff }}$ 's estimated above, bolometric corrections from Alonso et al. (1999), and the distance modulus for NGC 4833 from Harris (1996). We adopted a mass of $0.85 M_{\odot}$ for all stars and $M_{\mathrm{bol}, \odot}=4.75$ as the bolometric magnitude for the Sun, as in our previous studies.

We eliminated trends in the relation between abundances from Fe I lines and expected line strength (Magain 1984) to obtain values of the microturbulent velocity $v_{\mathrm{t}}$.

Finally, using the above values we interpolated within the Kurucz (1993) grid of model atmospheres (with the option for overshooting on) to derive the final abundances, adopting the model with the appropriate atmospheric parameters for each star. Its abundances matched those derived from Fe I lines. As discussed in Carretta et al. (2013a), this choice has a minimal impact on the derived abundances with respect to models without overshooting.

\subsection{Elemental abundances}

Most of our derived abundances rest on the analysis of equivalent widths (EW). The code ROSA (Gratton 1988) was used, as in previous papers, to measure EWs by adopting a relationship between EW and FWHM, as described in detail in Bragaglia et al. (2001). Following the approach used in Carretta et al. (2007b), we first corrected the EWs from GIRAFFE spectra to the system of the higher resolution UVES spectra, using seven stars observed with both instruments. The correction has the form $E W_{\mathrm{UVES}}=0.91( \pm 0.02) \times E W_{\mathrm{GIRAFFE}}+1.06( \pm 0.49)$ with an rms scatter of $5.4 \mathrm{~m} \AA$ and a Pearson correlation coefficient of $r=0.98$ from 121 lines.

Atomic parameters for the lines falling in the spectral range covered by UVES spectra and by setups HR11 and HR13 in the GIRAFFE spectra are comprehensively discussed in Gratton et al. (2003), together with the adopted solar reference abundances. 
Before co-adding, each HR13 GIRAFFE spectrum was corrected for blending with telluric lines due in particular to $\mathrm{H}_{2} \mathrm{O}$ and $\mathrm{O}_{2}$ near the forbidden [O I] line at $6300 \AA$, using synthetic spectra as described in Carretta et al. (2006). Corrections for any effects of departures from the local thermodynamical equilibrium (LTE) assumption according to the descriptions by Gratton et al. (1999) were applied to the derived $\mathrm{Na}$ abundances.

We derived abundances of $\mathrm{O}, \mathrm{Na}, \mathrm{Mg}$, and $\mathrm{Si}$ among the elements participating in the network of proton-capture reactions in H-burning at high temperature. Additionally, $\mathrm{Al}$ abundances were obtained from the doublet Al I 6696-98 $\AA$ for stars observed with UVES.

Besides $\mathrm{Mg}$ and $\mathrm{Si}$ (numbered among the proton-capture elements), we derived the abundance of the $\alpha$-elements Ca and Ti I, and $\mathrm{Ti}$ was also obtained from the singly ionized species in stars with UVES spectra. Abundances for the Fe-peak elements Sc II, V I, Cr I, Cr II, Mn I, Ni I, and Zn I were also derived, with abundances of some species only obtained for stars with UVES spectra, because of the larger spectral coverage. Corrections due to the hyperfine structure (references in Gratton et al. 2003) were applied to $\mathrm{Sc}, \mathrm{V}, \mathrm{Mn}$, and $\mathrm{Co}$. Abundances of $\mathrm{Cu} \mathrm{I}$ were derived from spectrum synthesis, as detailed in Carretta et al. (2011).

The concentration of the neutron-capture elements Y II, Ba II, La II, and Nd II was derived mostly for stars with UVES spectra and mostly from measurements of EWs. Results for Y and $\mathrm{Ba}$ were checked with synthetic spectra using line lists from D'Orazi et al. (2013) and D'Orazi et al. (2012), respectively. Abundances derived with the two methods are in very good agreement. Lanthanum abundances were obtained from EWs of three to four lines with transition parameters from Sneden et al. (2003; see also Carretta et al. 2011).

The available Ba lines are all very strong and quite sensitive to the velocity fields in the stellar atmospheres. As a consequence, a clear trend of $\mathrm{Ba}$ abundances as a function of the microturbulence results when using the values of $v_{\mathrm{t}}$ derived using the weaker Fe lines, formed typically in deeper atmospheric layers (see e.g. Carretta et al. 2013b, for a discussion of a similar effect in the analysis of NGC 362). To alleviate this problem, good results are obtained by adopting the values of $v_{\mathrm{t}}$ from the relation as a function of the surface gravity provided by Worley et al. (2013) for giants in the metal-poor GC M 15. When analysed using these values and a constant metallicity equal to the metal abundance derived for NGC 4833 (see next section), no trend is apparent, and we can safely explore the $\mathrm{Ba}$ abundances looking for intrinsic dispersion or correlations (if any) with other elements. The relation from Worley et al. was chosen since it was shown to efficiently remove any trend between $\mathrm{Ba}$ abundances and $v_{\mathrm{t}}$ for bright stars in a GC with metallicity comparable to NGC 4833. On average, the values of $v_{\mathrm{t}}$ from this relation are $0.26 \mathrm{~km} \mathrm{~s}^{-1}$ higher than the values derived as described in the previous section for individual stars (with rms $=0.29 \mathrm{~km} \mathrm{~s}^{-1}$, 78 stars). However, for all other species, the last method works very well, and we adopted, for all elements except $\mathrm{Ba}$, this approach that guarantees homogeneity with the more than $20 \mathrm{GCs}$ analysed by our group in this FLAMES survey.

\subsection{Metal abundances}

The mean metallicity we found for NGC 4833 from stars with high resolution UVES spectra is $[\mathrm{Fe} / \mathrm{H}]=-2.015 \pm 0.004 \pm$ $0.084 \mathrm{dex}$ ( $\mathrm{rms}=0.014 \mathrm{dex}, 12$ stars) from neutral species, where the first error is from statistics and the second refers to the systematic effects, as estimated in the next section. From

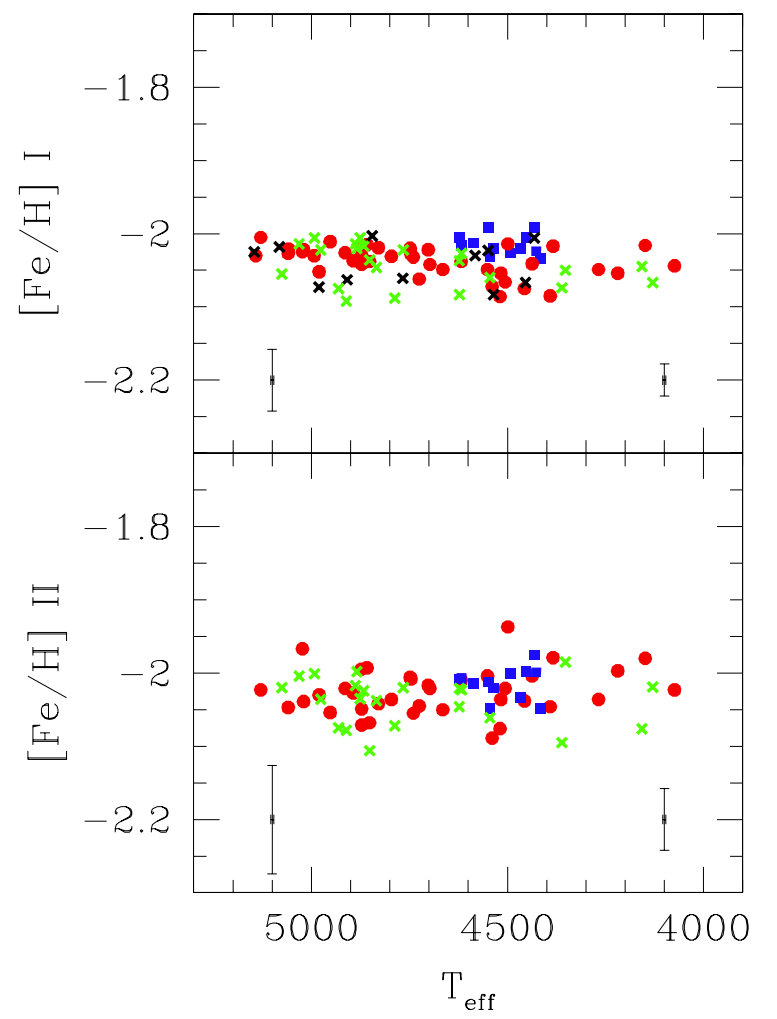

Fig. 4. Abundance ratios $[\mathrm{Fe} / \mathrm{H}]$ I (upper panel) and $[\mathrm{Fe} / \mathrm{H}]$ II (lower panel) as a function of $T_{\text {eff }}$ for all analysed stars. Blue squares are stars with UVES spectra, filled circles are those with GIRAFFE spectra observed with both the HR11 and HR13 setups, and crosses indicate stars observed with only the HR11 (black) or the HR13 (green) setup. Error bars on the right and on the left are star-to-star errors for targets observed with UVES and GIRAFFE, respectively.

the large sample of stars with GIRAFFE spectra, we derived a value of $[\mathrm{Fe} / \mathrm{H}]=-2.040 \pm 0.003 \pm 0.073 \mathrm{dex}(\mathrm{rms}=0.024 \mathrm{dex}$, 73 stars).

The abundances of iron obtained from the singly ionized species are in excellent agreement with those from neutral lines: $[\mathrm{Fe} / \mathrm{H}] \mathrm{II}=-2.014(\mathrm{rms}=0.021 \mathrm{dex}, 12$ stars $)$ from UVES and $[\mathrm{Fe} / \mathrm{H}] \mathrm{II}=-2.030(\mathrm{rms}=0.033 \mathrm{dex}, 59 \mathrm{stars})$ from GIRAFFE. The derived $\mathrm{Fe}$ abundances do not present any trend as a function of the effective temperature, as shown in Fig. 4.

Our average metal abundance seems to be about 0.2-0.3 dex lower than most of previous estimates in literature. From a preliminary analysis of RR Lyrae variables, Murray \& Darragh (2013) find $[\mathrm{Fe} / \mathrm{H}]=-1.67 \pm 0.13$ dex using Fourier decomposition of the light curves. We note, however, that the metallicities based on this method are often higher than those derived from high resolution spectroscopy, in particular in the low metallicity regime. For example, $[\mathrm{Fe} / \mathrm{H}]=-1.98$ dex from RR Lyrae in M 15 (Garcia Lugo et al. 2007), compared to [Fe/H] $=-2.32$ dex from high resolution spectroscopy (Carretta et al. 2009c); $[\mathrm{Fe} / \mathrm{H}]=-1.23$ dex for M 5 from RR Lyrae (Kaluzny et al. 2000) compared to -1.34 (Carretta et al. 2009c); $[\mathrm{Fe} / \mathrm{H}]=$ -2.11 dex from variables in M 30 (Kains et al. 2013), and -2.34 from Carretta et al. (2009c). The discrepancy is not limited to our group and analysis: Figuera Jaimes et al. (2013) derived $[\mathrm{Fe} / \mathrm{H}]=$ -1.64 from RR Lyraes in NGC 7492, while Cohen \& Melendez (2005) found -1.82 dex for this cluster from high resolution spectra. Using high resolution spectra and the MARCS models, Kraft and Ivans (2003) find $[\mathrm{Fe} / \mathrm{H}]=-2.33$ dex and $[\mathrm{Fe} / \mathrm{H}]=$ -2.39 dex for the metal-poor GCs M 30 and M 15, respectively. 
Table 3. Sensitivities of abundance ratios to variations in the atmospheric parameters, to errors in the equivalent widths (EWs), and errors in abundances for stars of NGC 4833 observed with UVES.

\begin{tabular}{lcccccccc}
\hline \hline Element & $\begin{array}{c}\text { Average } \\
n . \text { lines }\end{array}$ & $\begin{array}{c}T_{\text {eff }} \\
(\mathrm{K})\end{array}$ & $\begin{array}{c}\log g \\
(\mathrm{dex})\end{array}$ & $\begin{array}{c}{[\mathrm{A} / \mathrm{H}]} \\
(\mathrm{dex})\end{array}$ & $\begin{array}{c}v_{\mathrm{t}} \\
\mathrm{km} \mathrm{s}^{-1}\end{array}$ & $\begin{array}{c}E W \mathrm{~s} \\
(\mathrm{dex})\end{array}$ & $\begin{array}{c}\text { Total } \\
\text { internal }\end{array}$ & $\begin{array}{c}\text { Total } \\
\text { systematic }\end{array}$ \\
\hline Variation & & 50 & 0.20 & 0.10 & 0.10 & & & \\
internal & & 4 & 0.04 & 0.01 & 0.10 & 0.01 & & \\
systematic & & 57 & 0.06 & 0.08 & 0.03 & & & \\
\hline$[\mathrm{Fe} / \mathrm{H}] \mathrm{I}$ & 39 & +0.073 & -0.016 & -0.015 & -0.016 & 0.014 & 0.022 & 0.084 \\
{$[\mathrm{Fe} / \mathrm{H}] \mathrm{II}$} & 5 & -0.017 & +0.079 & +0.018 & -0.005 & 0.038 & 0.042 & 0.032 \\
{$[\mathrm{O} / \mathrm{Fe}] \mathrm{I}$} & 1 & -0.050 & +0.091 & +0.039 & +0.014 & 0.085 & 0.088 & 0.090 \\
{$[\mathrm{Na} / \mathrm{Fe}] \mathrm{I}$} & 2 & -0.038 & -0.030 & -0.003 & +0.011 & 0.060 & 0.061 & 0.096 \\
{$[\mathrm{Mg} / \mathrm{Fe}] \mathrm{I}$} & 1 & -0.029 & +0.000 & +0.002 & +0.011 & 0.085 & 0.086 & 0.072 \\
{$[\mathrm{Al} / \mathrm{Fe}] \mathrm{I}$} & 1 & -0.034 & +0.002 & +0.003 & +0.013 & 0.085 & 0.086 & 0.105 \\
{$[\mathrm{Si} / \mathrm{Fe}] \mathrm{I}$} & 4 & -0.050 & +0.025 & +0.011 & +0.013 & 0.043 & 0.045 & 0.059 \\
{$[\mathrm{Ca} / \mathrm{Fe}] \mathrm{I}$} & 15 & -0.016 & -0.006 & -0.003 & +0.003 & 0.022 & 0.022 & 0.018 \\
{$[\mathrm{Sc} / \mathrm{Fe}] \mathrm{II}$} & 6 & +0.011 & -0.007 & +0.003 & +0.011 & 0.035 & 0.035 & 0.029 \\
{$[\mathrm{Ti} / \mathrm{Fe}] \mathrm{I}$} & 5 & +0.019 & -0.005 & -0.002 & -0.014 & 0.038 & 0.040 & 0.014 \\
{$[\mathrm{Ti} / \mathrm{Fe}] \mathrm{II}$} & 9 & +0.025 & -0.013 & -0.002 & -0.002 & 0.028 & 0.032 & 0.023 \\
{$[\mathrm{~V} / \mathrm{Fe}] \mathrm{II}$} & 2 & +0.016 & -0.006 & -0.002 & +0.013 & 0.060 & 0.062 & 0.019 \\
{$[\mathrm{Cr} / \mathrm{Fe}] \mathrm{I}$} & 9 & +0.013 & -0.011 & -0.007 & -0.009 & 0.028 & 0.030 & 0.017 \\
{$[\mathrm{Cr} / \mathrm{Fe}] \mathrm{II}$} & 4 & -0.001 & -0.011 & -0.009 & +0.000 & 0.043 & 0.043 & 0.013 \\
{$[\mathrm{Mn} / \mathrm{Fe}] \mathrm{I}$} & 2 & -0.012 & -0.000 & +0.001 & +0.014 & 0.060 & 0.062 & 0.015 \\
{$[\mathrm{Co} / \mathrm{Fe}] \mathrm{I}$} & 1 & -0.014 & +0.001 & +0.003 & +0.013 & 0.085 & 0.086 & 0.020 \\
{$[\mathrm{Ni} / \mathrm{Fe}] \mathrm{I}$} & 8 & +0.005 & +0.008 & +0.003 & +0.007 & 0.030 & 0.031 & 0.007 \\
{$[\mathrm{Cu} / \mathrm{Fe}] \mathrm{II}$} & 1 & +0.010 & +0.003 & +0.001 & +0.010 & 0.085 & 0.086 & 0.021 \\
{$[\mathrm{Zn} / \mathrm{Fe}] \mathrm{II}$} & 1 & -0.075 & +0.056 & +0.022 & -0.001 & 0.085 & 0.085 & 0.088 \\
{$[\mathrm{Y} / \mathrm{Fe}] \mathrm{II}$} & 9 & +0.031 & -0.012 & +0.000 & -0.013 & 0.028 & 0.031 & 0.040 \\
{$[\mathrm{Ba} / \mathrm{Fe}] \mathrm{II}$} & 3 & +0.043 & -0.010 & +0.002 & -0.075 & 0.049 & 0.090 & 0.065 \\
{$[\mathrm{La} / \mathrm{Fe}] \mathrm{II}$} & 3 & +0.040 & -0.009 & +0.003 & +0.001 & 0.049 & 0.049 & 0.046 \\
{$[\mathrm{Nd} / \mathrm{Fe}] \mathrm{II}$} & 4 & +0.041 & -0.009 & +0.003 & -0.000 & 0.043 & 0.043 & 0.048 \\
\hline & & & & & & & & \\
\hline & & & & & & & \\
& & & & & & & &
\end{tabular}

From CCD photometry, Melbourne et al. (2000) derived a mean metallicity $[\mathrm{Fe} / \mathrm{H}]=-1.83 \pm 0.14$ dex (and a reddening $E(B-V)=0.32 \pm 0.03)$. Early detections based on high resolution spectra also obtained similar high values: $[\mathrm{Fe} / \mathrm{H}]=$ -1.71 dex from one star (Minniti et al. 1996) and $[\mathrm{Fe} / \mathrm{H}]=$ -1.74 dex, on average, from two stars (Gratton \& Ortolani 1989). Moreover, Kraft and Ivans (2003) found for this GC a metallicity $[\mathrm{Fe} / \mathrm{H}]=-2.06,-2.00,-2.04$ dex from MARCS, and Kurucz models with and without the overshooting option, respectively.

\subsection{Error budget}

Our procedure for error estimates is amply described in Carretta et al. (2007b, 2009a,b) so will not be repeated here. In the following we only provide the main tables for the sensitivities of abundance ratios to the adopted errors in the atmospheric parameters and EWs and the final estimates of internal and systematic errors for all species analysed from the UVES and GIRAFFE spectra of stars in NGC 4833.

The sensitivities of derived abundances on the adopted atmospheric parameters were obtained by repeating our abundance analysis by changing only one atmospheric parameter each time for all stars in NGC 4833 (separately for the UVES and the GIRAFFE samples). The sensitivity in each parameter was adopted as the one corresponding to the average of all the sample.

The amount of the change in the input parameters $T_{\text {eff }}, \log g$, $[\mathrm{A} / \mathrm{H}]$, and $v_{\mathrm{t}}$ to compute the sensitivity of abundances to variations in the atmospheric parameters is shown in the first line of the headers in Tables 3 and 4, whereas the resulting response in abundance changes of all elements (the sensitivities) are shown in columns from 3 to 6 of these tables.
The averages of all measured elements with their rms scatter are listed in Table 5. Derived atmospheric parameters and Fe abundances for individual stars in NGC 4833 are in Table 6; abundances of proton-capture, $\alpha$-capture, Fe-peak, and neutroncapture elements are provided in Tables 7-11, respectively. These tables are only available at the CDS: a few lines are given for guidance. Upon request by the referee, we also report the average and rms scatter of the $[\mathrm{Na} / \mathrm{Fe}]$ ratio in LTE for individual stars in the last two columns of Table 7, to give an idea of the adopted NLTE corrections.

\section{Results}

\subsection{The Na-O anticorrelation in NGC 4833}

After combining the UVES and GIRAFFE datasets and taking into account stars observed with both instruments, we ended with 61 stars with $\mathrm{O}$ abundances (40 actual detections and 21 upper limits) and 60 stars with $\mathrm{Na}$ abundances. The Na-O anticorrelation in NGC 4833 rests on 51 giants with both $\mathrm{O}$ and $\mathrm{Na}$, and is shown in Fig. 5, with star-to-star errors relative to the GIRAFFE dataset. Internal error bars for the UVES sample are slightly smaller (see Table 3 ).

It is not easy to judge whether stars in NGC 4833 are grouped into discrete populations with homogeneous composition, along the Na-O anti-correlation, which is mostly formed with the stars observed with GIRAFFE. Similar large samples are better suited to quantifying the extension of this feature, but the associated internal errors may smear possible groups, although some subdivisions are recognizable in Fig. 5. The limited sample of giants observed with UVES seems to be better suited to this task.

The 12 stars with UVES spectra are clearly clustered in two clearly separated groups: one with abundances typical of the pattern established by core-collapse supernovae nucleosynthesis 
Table 4. Sensitivities of abundance ratios to variations in the atmospheric parameters, to errors in the EWs, and errors in abundances for stars of NGC 4833 observed with GIRAFFE.

\begin{tabular}{lcccccccc}
\hline \hline Element & $\begin{array}{c}\text { Average } \\
n . \text { lines }\end{array}$ & $\begin{array}{c}T_{\text {eff }} \\
(\mathrm{K})\end{array}$ & $\begin{array}{c}\log g \\
(\mathrm{dex})\end{array}$ & $\begin{array}{c}{[\mathrm{A} / \mathrm{H}]} \\
(\mathrm{dex})\end{array}$ & $\begin{array}{c}v_{\mathrm{t}} \\
\mathrm{km} \mathrm{s}^{-1}\end{array}$ & $\begin{array}{c}E W \mathrm{~s} \\
(\mathrm{dex})\end{array}$ & $\begin{array}{c}\text { Total } \\
\text { internal }\end{array}$ & $\begin{array}{c}\text { Total } \\
\text { systematic }\end{array}$ \\
\hline Variation & & 50 & 0.20 & 0.10 & 0.10 & & & \\
internal & & 4 & 0.04 & 0.02 & 0.22 & 0.02 & & \\
systematic & & 57 & 0.06 & 0.07 & 0.03 & & & \\
\hline$[\mathrm{Fe} / \mathrm{H}] \mathrm{I}$ & 20 & +0.064 & -0.011 & -0.011 & -0.016 & 0.023 & 0.042 & 0.073 \\
{$[\mathrm{Fe} / \mathrm{H}] \mathrm{II}$} & 2 & -0.016 & +0.077 & +0.011 & -0.004 & 0.071 & 0.074 & 0.030 \\
{$[\mathrm{O} / \mathrm{Fe}] \mathrm{I}$} & 1 & -0.040 & +0.085 & +0.031 & +0.018 & 0.101 & 0.110 & 0.066 \\
{$[\mathrm{Na} / \mathrm{Fe}] \mathrm{I}$} & 2 & -0.034 & -0.026 & +0.004 & +0.013 & 0.071 & 0.077 & 0.055 \\
{$[\mathrm{Mg} / \mathrm{Fe}] \mathrm{I}$} & 1 & -0.027 & +0.001 & +0.002 & +0.013 & 0.101 & 0.105 & 0.039 \\
{$[\mathrm{Si} / \mathrm{Fe}] \mathrm{I}$} & 3 & -0.046 & +0.021 & +0.009 & +0.015 & 0.058 & 0.067 & 0.053 \\
{$[\mathrm{Ca} / \mathrm{Fe}] \mathrm{I}$} & 4 & -0.015 & -0.006 & -0.001 & +0.001 & 0.051 & 0.051 & 0.017 \\
{$[\mathrm{Sc} / \mathrm{Fe}] \mathrm{II}$} & 4 & -0.052 & +0.082 & +0.026 & +0.011 & 0.051 & 0.059 & 0.064 \\
{$[\mathrm{Ti} / \mathrm{Fe}] \mathrm{I}$} & 3 & +0.004 & -0.004 & +0.001 & +0.015 & 0.058 & 0.067 & 0.007 \\
{$[\mathrm{~V} / \mathrm{Fe}] \mathrm{I}$} & 3 & +0.021 & -0.008 & -0.003 & +0.020 & 0.058 & 0.073 & 0.025 \\
{$[\mathrm{Cr} / \mathrm{Fe}] \mathrm{I}$} & 1 & +0.011 & -0.006 & -0.001 & +0.021 & 0.101 & 0.111 & 0.018 \\
{$[\mathrm{Co} / \mathrm{Fe}] \mathrm{I}$} & 1 & -0.001 & +0.003 & +0.005 & +0.023 & 0.101 & 0.113 & 0.015 \\
{$[\mathrm{Ni} / \mathrm{Fe}] \mathrm{I}$} & 3 & -0.001 & +0.008 & +0.004 & +0.013 & 0.058 & 0.065 & 0.006 \\
{$[\mathrm{Ba} / \mathrm{Fe}] \mathrm{II}$} & 1 & -0.033 & +0.077 & +0.024 & -0.067 & 0.101 & 0.179 & 0.056 \\
\hline
\end{tabular}

Table 5. Mean abundances from UVES and GIRAFFE.

\begin{tabular}{lrcc}
\hline \hline Element & \multicolumn{2}{c}{ UVES } & \multicolumn{2}{c}{ GIRAFFE } \\
& $n$ avg rms & \multicolumn{2}{c}{$n$ avg rms } \\
\hline$[\mathrm{O} / \mathrm{Fe}] \mathrm{I}$ & $12+0.170 .22$ & $51+0.250 .29$ \\
{$[\mathrm{Na} / \mathrm{Fe}] \mathrm{I}$} & $12+0.520 .29$ & $52+0.460 .27$ \\
{$[\mathrm{Mg} / \mathrm{Fe}] \mathrm{I}$} & $12+0.270 .22$ & $44+0.360 .15$ \\
{$[\mathrm{Al} / \mathrm{Fe}] \mathrm{I}$} & $12+0.900 .34$ & \\
{$[\mathrm{Si} / \mathrm{Fe}] \mathrm{I}$} & $12+0.470 .04$ & $66+0.460 .05$ \\
{$[\mathrm{Ca} / \mathrm{Fe}] \mathrm{I}$} & $12+0.350 .01$ & $73+0.350 .02$ \\
{$[\mathrm{Sc} / \mathrm{Fe}] \mathrm{II}$} & $12-0.040 .01$ & $73-0.040 .02$ \\
{$[\mathrm{Ti} / \mathrm{Fe}] \mathrm{I}$} & $12+0.180 .02$ & $53+0.170 .02$ \\
{$[\mathrm{Ti} / \mathrm{Fe}] \mathrm{II}$} & $12+0.230 .01$ & \\
{$[\mathrm{~V} / \mathrm{Fe}] \mathrm{I}$} & $12-0.080 .01$ & $24-0.100 .02$ \\
{$[\mathrm{Cr} / \mathrm{Fe}] \mathrm{I}$} & $12-0.240 .02$ & $24-0.200 .04$ \\
{$[\mathrm{Cr} / \mathrm{Fe}] \mathrm{II}$} & $12+0.010 .05$ & \\
{$[\mathrm{Mn} / \mathrm{Fe}] \mathrm{I}$} & $12-0.540 .01$ & \\
{$[\mathrm{Fe} / \mathrm{H}] \mathrm{I}$} & $12-2.020 .01$ & $73-2.040 .02$ \\
{$[\mathrm{Fe} / \mathrm{H}] \mathrm{II}$} & $12-2.010 .02$ & $59-2.030 .03$ \\
{$[\mathrm{Co} / \mathrm{Fe}] \mathrm{I}$} & $8-0.030 .03$ & $7-0.070 .04$ \\
{$[\mathrm{Ni} / \mathrm{Fe}] \mathrm{I}$} & $12-0.180 .01$ & $68-0.180 .03$ \\
{$[\mathrm{Cu} / \mathrm{Fe}] \mathrm{I}$} & $12-0.800 .09$ & \\
{$[\mathrm{Zn} / \mathrm{Fe}] \mathrm{I}$} & $12+0.070 .03$ & \\
{$[\mathrm{Y} / \mathrm{Fe}] \mathrm{II}$} & $12-0.150 .06$ & \\
{$[\mathrm{Ba} / \mathrm{Fe}] \mathrm{II}$} & $12-0.060 .07$ & $62-0.200 .14$ \\
{$[\mathrm{La} / \mathrm{Fe}] \mathrm{II}$} & $12+0.050 .03$ & \\
{$[\mathrm{Nd} / \mathrm{Fe}] \mathrm{II}$} & $12+0.420 .04$ & \\
\hline & &
\end{tabular}

(high $\mathrm{O}$ and low $\mathrm{Na}$ ), also shared by field stars of similar metallicity (e.g. Gratton et al. 2000), the other with low-O/high-Na abundances, whose counterparts are rarely observed in Galactic field stars. To check that this occurrence in NGC 4833 is not a spurious effect due to the limited number of giants in the UVES sample, we plotted in Fig. 6 (upper panel) the distribution of the $[\mathrm{O} / \mathrm{Na}]$ ratios from our total sample of about $250 \mathrm{RGB}$ stars observed with UVES in 22 GCs of the Milky Way in our FLAMES survey. The colour coding corresponds to the division of stars into the primordial $(\mathrm{P})$ component of first generation stars, and to the two fractions of stars with intermediate (I) and extreme (E) composition within the second stellar generation in GCs, as defined in Carretta et al. (2009a) from their location along the $\mathrm{Na}-\mathrm{O}$ anticorrelation. Our UVES sample in NGC 4833 (shown in Fig. 6 with number counts multiplied by a factor 3 to improve clarity) clearly splits into two groups, roughly coincident with the first and second generation stars. As a comparison, in the lower panel we also plot the only other large sample in an individual GC based on high resolution spectra, the about 100 RGB stars observed in M 4 by Marino et al. (2008). An offset of 0.1 dex was arbitrarily subtracted to their $[\mathrm{O} / \mathrm{Na}$ ] values to bring them on our abundance scale. In M 4, with its short anticorrelation, the extreme component of second-generation stars is obviously not present.

Using the quantitative criteria introduced by Carretta et al. (2009a), we can use $\mathrm{O}$ and $\mathrm{Na}$ abundances to quantify the fraction of the different stellar generations. From the total sample of 51 stars with $\mathrm{O}$ and $\mathrm{Na}$, we found that the fractions of $\mathrm{P}, \mathrm{I}$, and E stars for NGC 4833 are $31 \pm 8 \%, 59 \pm 11 \%$, and $10 \pm 4 \%$, respectively. The fraction of first-generation stars is similar to the one (about one third) typical of the overwhelming majority of Galactic GCs. On the other hand, the fraction of secondgeneration $\mathrm{E}$ stars with extremely modified composition is quite large in NGC 4833. As a comparison, the $\mathrm{E}$ fraction in $\mathrm{GCs}$ like NGC 4590 (M 68), NGC 6809 (M 55), NGC 7078 (M 15), NGC 7099 (M 30), bracketing NGC 4833 in mass and metallicity, does not exceed $2 \%$ or $3 \%$ (and it is formally absent in M 15 and M 68, Carretta et al. 2009a,b). Among the observed RGB stars in NGC 4833, there is apparently no statistically significant segregation in radial distance from the cluster centre for the P, I, and E components. Our sample of member stars is, however, all confined within two half-mass radii and may not be the optimal sample for this kind of analysis. Large photometric databases are better suited to studying possible difference of radial concentration of different stellar generations.

The interquartile range (IQR) for the ratio $[\mathrm{O} / \mathrm{Na}]$ is a very useful measurement to quantify the extension of the Na-O anticorrelation (Carretta 2006). From our large sample we found that $\mathrm{IQR}[\mathrm{O} / \mathrm{Na}]=0.945 \mathrm{dex}$ in NGC 4833. Therefore, this cluster joins the ensemble of other GCs producing a nice correlation with the cluster total mass (represented by the proxy of the total absolute magnitude, $M_{V}=-8.16$ for NGC 4833 from Harris 1996), established in Carretta et al. (2010a) and reproduced in the left-hand panel of Fig. 7. The location of NGC 4833 in this plot seems to be in the upper envelope of the relation defined by 


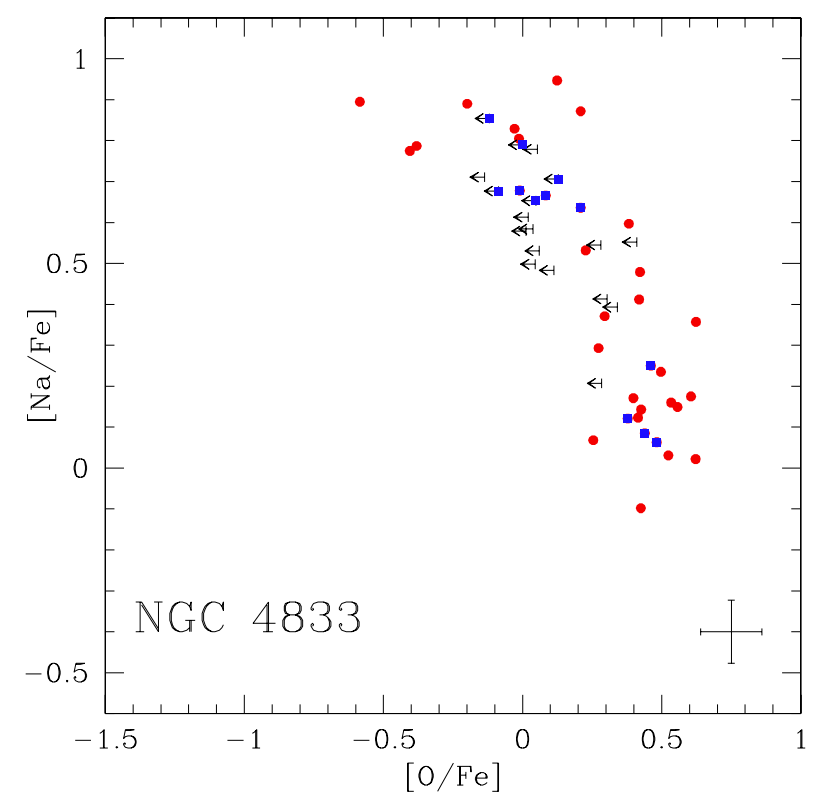

Fig. 5. Na-O anti-correlation observed in NGC 4833. Blue squares are stars observed with UVES, while red circles indicate stars with GIRAFFE spectra. Upper limits in $\mathrm{O}$ are shown as arrows, and starto-star (internal) error bars are plotted.

the bulk of the other GCs. The position of another cluster sharing a similar position (NGC 288) is also indicated. This occurrence is discussed in Sect. 5.

Recio-Blanco et al. (2006) computed the maximum temperature reached along the horizontal branch (HB) in NGC 4833: $\log T_{\text {eff }}=4.301$. The correlation between this parameter and the extension of the $\mathrm{Na}-\mathrm{O}$ anticorrelation, discovered by Carretta et al. (2007c), is updated and shown in the righthand panel of Fig. 7. Once again, NGC 4833 seems to lie at the upper envelope of the relation.

\subsection{Other proton-capture elements}

Apart from the case of $\mathrm{O}$ and $\mathrm{Na}$, significant star-to-star abundance variations are detected for other proton-capture elements in giants in NGC 4833. In particular, we found that the $[\mathrm{Mg} / \mathrm{Fe}]$ abundance ratio shows an unusually wide spread in this cluster, with peak-to-peak variations of more than $0.5 \mathrm{dex}$.

The reality of the intrinsic scatter in $\mathrm{Mg}$ is immediately evident when comparing the estimated internal error for the UVES sample (0.086 dex) to the observed rms scatter in $[\mathrm{Mg} / \mathrm{Fe}]$ ( 0.223 dex): the cosmic spread in $\mathrm{Mg}$ among RGB stars in NGC 4833 is significant at almost a $3 \sigma$ level.

The run of $[\mathrm{Mg} / \mathrm{Fe}]$ ratios as a function of the abundance of the proton-capture elements $\mathrm{O}, \mathrm{Na}$, and $\mathrm{Si}$ is shown in Fig. 8, together with the classical $\mathrm{Na}-\mathrm{O}$ anticorrelation. The error bars indicate star-to-star errors and refer to the GIRAFFE sample; internal errors for stars in the UVES sample are usually smaller (see Table 3). The Mg abundance is correlated to that of $\mathrm{O}$ and anti-correlated with species enhanced in the network of protoncapture reactions, namely $\mathrm{Na}$ and $\mathrm{Si}$. We retrieved this pattern from both the datasets observed with UVES and with GIRAFFE.

In the case of the GIRAFFE sample, the observed spread in $\mathrm{Mg}(0.151 \mathrm{dex})$ does not formally exceed the associated internal error $(0.105$ dex $)$ too much, most likely thanks to the lower resolution of the spectra and the extension of the GIRAFFE sample to warmer giants, with weaker lines. However, even in

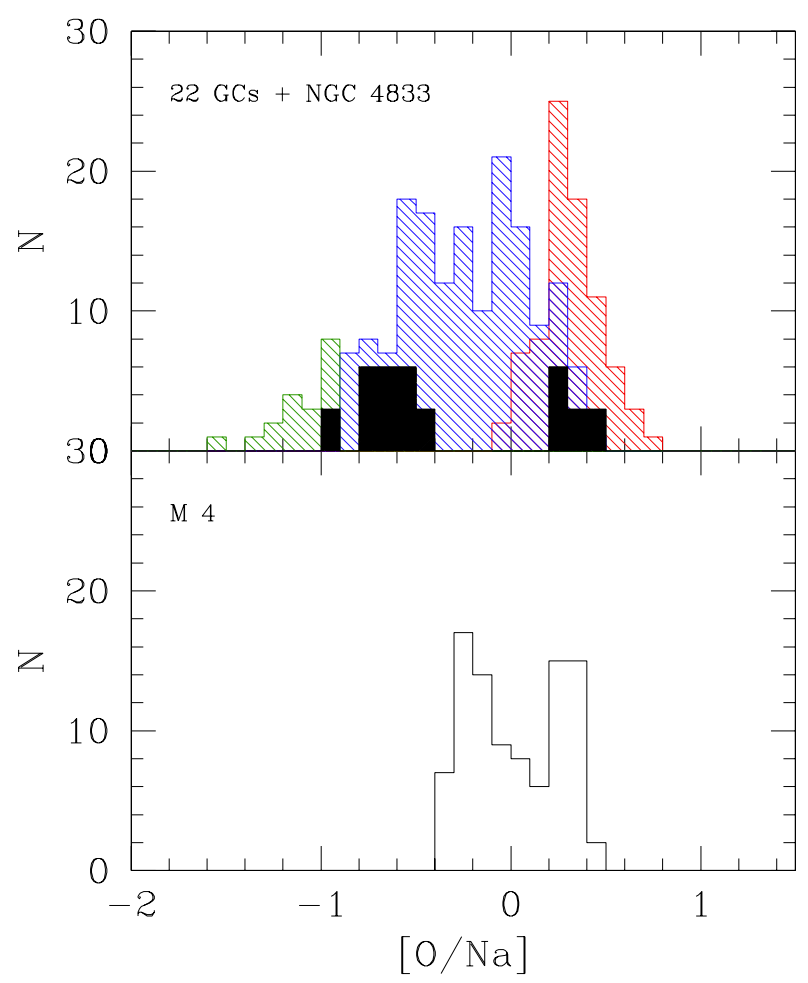

Fig. 6. Distribution of the $[\mathrm{O} / \mathrm{Na}]$ abundance ratios in several GCs from stars observed with the high resolution UVES spectrograph. In the upper panel, about 250 RGB stars in 23 GCs from our FLAMES survey are plotted. Colour coding is red, blue, and green for the P, I, and E components defined in Carretta et al. (2009a). In black we plotted the distribution for NGC 4833 from the present work, with the numbers of stars multiplied by 3 to improve the visibility. Lower panel: distribution of $[\mathrm{O} / \mathrm{Na}$ ] from UVES spectra of about 100 giants in $\mathrm{M} 4$ from Marino et al. (2008).

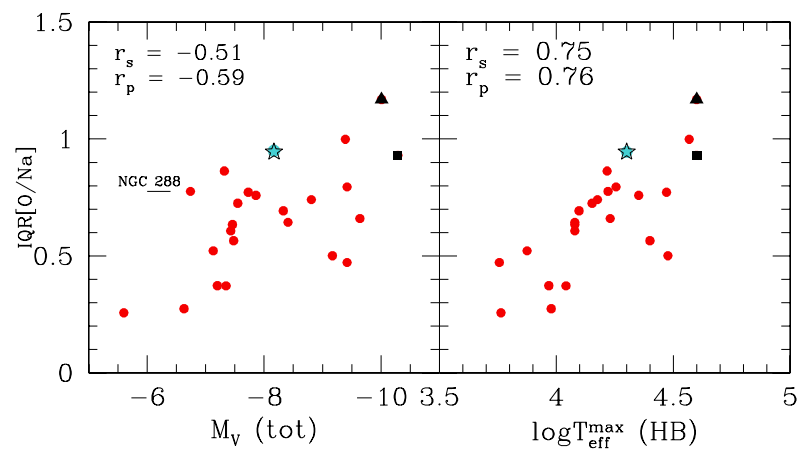

Fig. 7. IQR[O/Na] ratios for NGC 4833 (star symbol) and other Galactic GCs as a function of the total cluster absolute magnitude $M_{V}$ (left panel). The other clusters are $\omega$ Cen (filled square, Johnson and Pilachowski 2010), M 54 (filled triangle, Carretta et al. 2010b), and other GCs from our FLAMES survey (filled circles, Carretta et al. 2009a, 2011, 2013b). In the right panel the IQR[O/Na] is shown as a function of the maximum temperature on the horizontal branch, from Recio-Blanco et al. (2006). In each panel, the Spearman rank correlation coefficient $\left(r_{\mathrm{s}}\right)$ and the Pearson's correlation coefficient $\left(r_{\mathrm{p}}\right)$ are reported.

this case there is no doubt that $\mathrm{Mg}$ variations in NGC 4833 are real. In Fig. 9 the HR11 spectra of the two stars with the lowest $\mathrm{Mg}$ abundances in the GIRAFFE sample (star 36391, with $[\mathrm{Mg} / \mathrm{Fe}]=+0.02$ dex, and $\operatorname{star} 34613$ with $[\mathrm{Mg} / \mathrm{Fe}]=0.00)$ are compared to the spectra of two other stars with similar 
Table 6. Adopted atmospheric parameters and derived iron abundances.

\begin{tabular}{lcccccccccc}
\hline \hline Star & $\begin{array}{c}T_{\text {eff }} \\
(\mathrm{K})\end{array}$ & $\begin{array}{c}\log g \\
(\mathrm{dex})\end{array}$ & $\begin{array}{c}{[\mathrm{A} / \mathrm{H}]} \\
(\mathrm{dex})\end{array}$ & $\begin{array}{c}v_{\mathrm{t}} \\
\left(\mathrm{km} \mathrm{s}^{-1}\right)\end{array}$ & $\begin{array}{c}{[\mathrm{Fe} / \mathrm{H}] \mathrm{I}} \\
(\mathrm{dex})\end{array}$ & rms & $n r$ & $\begin{array}{c}{[\mathrm{Fe} / \mathrm{HII}} \\
(\mathrm{dex})\end{array}$ & $\mathrm{rms}$ \\
\hline 22810 & 4893 & 2.17 & -2.04 & 1.46 & 23 & -2.037 & 0.118 & 2 & -2.027 & 0.115 \\
23306 & 4914 & 2.20 & -2.03 & 1.44 & 21 & -2.026 & 0.140 & 1 & -2.021 & \\
23437 & 4767 & 1.89 & -2.06 & 1.19 & 5 & -2.061 & 0.062 & & & \\
23491 & 4074 & 0.45 & -2.04 & 2.07 & 38 & -2.044 & 0.105 & 3 & -2.023 & 0.037 \\
23518 & 4867 & 2.12 & -2.02 & 1.43 & 20 & -2.017 & 0.101 & 2 & -2.024 & 0.001 \\
24063 & 4352 & 1.04 & -2.05 & 1.88 & 26 & -2.050 & 0.090 & 2 & -1.985 & 0.005 \\
24252 & 4432 & 1.20 & -1.99 & 1.88 & 38 & -1.992 & 0.058 & 5 & -1.975 & 0.040 \\
\hline
\end{tabular}

Notes. The complete table is available is at the CDS.

Table 7. Abundances of proton-capture elements in stars of NGC 4833.

\begin{tabular}{|c|c|c|c|c|c|c|c|c|c|c|c|c|c|c|c|c|}
\hline Star & $n$ & {$[\mathrm{O} / \mathrm{Fe}]$} & $\mathrm{rms}$ & $n$ & {$[\mathrm{Na} / \mathrm{Fe}]$} & $\mathrm{rms}$ & $n$ & {$[\mathrm{Mg} / \mathrm{Fe}]$} & $\mathrm{rms}$ & $n$ & {$[\mathrm{Al} / \mathrm{Fe}]$} & $\mathrm{rms}$ & $\operatorname{limO}$ & $\lim A 1$ & {$[\mathrm{Na} / \mathrm{Fe}]_{\mathrm{LTE}}$} & $\mathrm{rms}$ \\
\hline 22810 & 1 & +0.23 & & 3 & +0.53 & 0.04 & 1 & +0.34 & & & & & 1 & & +0.49 & 0.05 \\
\hline 23306 & 1 & +0.62 & & 2 & +0.36 & 0.10 & 2 & +0.59 & 0.03 & & & & 1 & & +0.28 & 0.11 \\
\hline 23437 & & & & 2 & +0.36 & 0.03 & 1 & +0.39 & & & & & 1 & & +0.22 & 0.03 \\
\hline 23491 & 1 & -0.01 & & 3 & +0.80 & 0.05 & 2 & +0.25 & 0.02 & & & & 1 & & +0.38 & 0.05 \\
\hline 23518 & 1 & +0.55 & & & & & 1 & +0.47 & & & & & 1 & & & \\
\hline 24063 & 2 & -0.20 & 0.03 & 2 & +0.89 & 0.00 & & & & & & & 1 & & +0.58 & 0.00 \\
\hline 24252 & 1 & +0.48 & & 2 & +0.06 & 0.02 & 1 & +0.58 & & 1 & +0.43 & & 1 & 0 & -0.12 & 0.02 \\
\hline
\end{tabular}

Notes. $n$ is the number of lines used in the analysis. Upper limits (limO, $\mathrm{Al}=0$ ) and detections $(=1)$ for $\mathrm{O}$ and $\mathrm{Al}$ are flagged. The complete table is at the CDS.
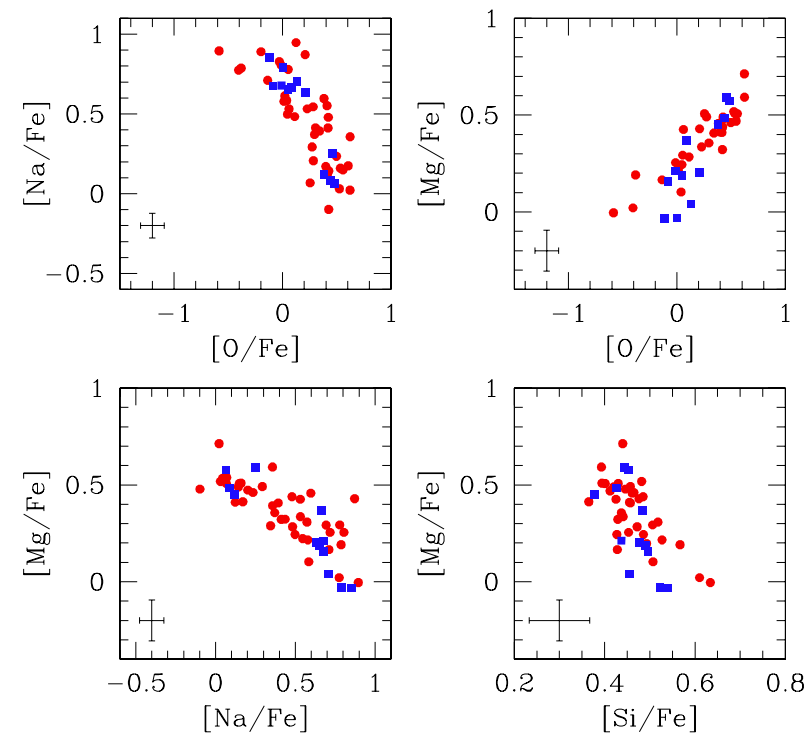

Fig. 8. Relations among proton-capture elements $\mathrm{O}, \mathrm{Na}, \mathrm{Mg}$, and $\mathrm{Si}$ in NGC 4833. Red circles refer to stars with GIRAFFE spectra, blue squares indicate stars observed with UVES. The internal errorbars plotted in each panel are those relative to the GIRAFFE sample.

atmospheric parameters, but quite different (much higher) $\mathrm{Mg}$ abundances.

This direct comparison, free of any uncertainties related to the abundance analysis, robustly corroborates our findings: there is a wide spread in Mg in NGC 4833, where giants with a solar $[\mathrm{Mg} / \mathrm{Fe}]$ ratio stand side-by-side with stars having a normal $[\mathrm{Mg} / \mathrm{Fe}]$ ratio appropriate for metal-poor halo stars.

Large depletions of $\mathrm{Mg}$ due to the action of proton-capture reactions in H-burning at high temperature should have two main consequences: produce $\mathrm{Al}$ through the $\mathrm{Mg}$-Al cycle and, if the burning temperatures are high enough, slightly enhance

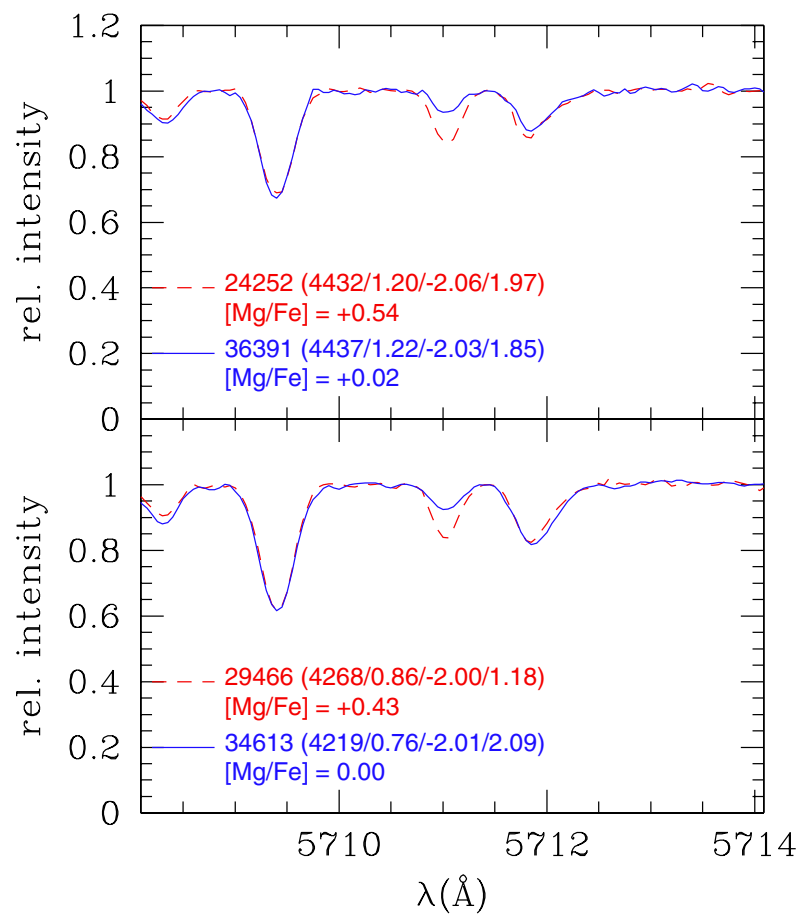

Fig. 9. Comparison of two pairs of Mg-poor and Mg-normal stars with similar atmospheric parameters in the Mg I $5711.09 \AA$ spectral region. Atmospheric parameters $T_{\text {eff }}, \log g$, metallicity, $v_{\mathrm{t}}$, and the $[\mathrm{Mg} / \mathrm{Fe}]$ abundance ratios of stars are indicated in each panel.

the abundance of Si through the mechanism of the leakage from the $\mathrm{Mg}$-Al cycle on ${ }^{28} \mathrm{Si}$ (Karakas \& Lattanzio 2003).

Abundances of Al can be obtained from the UVES spectra, but in four out of 12 stars observed with UVES only upper limits could be derived. Nevertheless, the derived values do not show any trend as a function of the effective temperature, and their 
Table 8. Abundances of $\alpha$-elements in stars of NGC 4833.

\begin{tabular}{lcccccccccccc}
\hline \hline Star & $n$ & {$[\mathrm{Si} / \mathrm{Fe}]$} & $\mathrm{rms}$ & $n$ & {$[\mathrm{Ca} / \mathrm{Fe}]$} & $\mathrm{rms}$ & $n$ & {$[\mathrm{Ti} / \mathrm{Fe}] \mathrm{I}$} & rms & $n$ & {$[\mathrm{Ti} / \mathrm{Fe}] \mathrm{II}$} & $\mathrm{rms}$ \\
\hline 22810 & 6 & +0.44 & 0.22 & 4 & +0.36 & 0.05 & 1 & +0.19 & & & & \\
23306 & 2 & +0.39 & 0.01 & 6 & +0.35 & 0.09 & 2 & +0.17 & 0.09 & & & \\
23437 & 2 & +0.51 & 0.06 & 1 & +0.31 & & & & & & & \\
23491 & 6 & +0.45 & 0.14 & 5 & +0.34 & 0.12 & 4 & +0.15 & 0.05 & & & \\
23518 & 1 & +0.41 & & 3 & +0.38 & 0.02 & 1 & +0.17 & & & & \\
24063 & 1 & +0.40 & & 5 & +0.37 & 0.05 & 3 & +0.17 & 0.01 & & & \\
24252 & 5 & +0.45 & 0.06 & 13 & +0.35 & 0.05 & 7 & +0.17 & 0.06 & 8 & +0.22 & 0.04 \\
\hline
\end{tabular}

Notes. $n$ is the number of lines used in the analysis. The complete table is at the CDS.

Table 9. Abundances of Fe-peak elements in stars of NGC 4833.

\begin{tabular}{|c|c|c|c|c|c|c|c|c|c|}
\hline Star & $n[\mathrm{Sc} / \mathrm{Fe}] \mathrm{II} \mathrm{rms}$ & $n[\mathrm{~V} / \mathrm{Fe}] \mathrm{rms}$ & $n[\mathrm{Cr} / \mathrm{Fe}] \mathrm{I} \mathrm{rms}$ & $n[\mathrm{Cr} / \mathrm{Fe}] \mathrm{II} \mathrm{rms}$ & $n[\mathrm{Mn} / \mathrm{Fe}] \mathrm{rms}$ & $n[\mathrm{Co} / \mathrm{Fe}] \mathrm{rms}$ & $n[\mathrm{Ni} / \mathrm{Fe}] \mathrm{rms}$ & $n[\mathrm{Cu} / \mathrm{Fe}] \mathrm{rms}$ & $n[\mathrm{Zn} / \mathrm{Fe}] \mathrm{I} \mathrm{rms}$ \\
\hline 22810 & $4-0.040 .09$ & & & & & & $3-0.160 .27$ & & \\
\hline 23306 & $\begin{array}{lll}5 & -0.03 & 0.24\end{array}$ & & & & & & $2-0.230 .19$ & & \\
\hline 23437 & $5-0.060 .06$ & & & & & & $2-0.150 .18$ & & \\
\hline 23491 & $6+0.000 .04$ & $4-0.110 .03$ & $2-0.210 .10$ & & & $1-0.09$ & $6-0.160 .11$ & & \\
\hline 23518 & $1-0.03$ & & & & & & $2-0.190 .06$ & & \\
\hline 24063 & $2-0.050 .08$ & $2-0.090 .05$ & $1-0.20$ & & & & $3-0.160 .06$ & & \\
\hline 24252 & $8-0.050 .14$ & $4-0.100 .01$ & $12-0.210 .07$ & $6+0.010 .08$ & $2-0.540 .08$ & $1-0.05$ & $\begin{array}{lll}9 & -0.17 & 0.07\end{array}$ & $1-0.85$ & $1+0.04$ \\
\hline
\end{tabular}

Notes. $n$ is the number of lines used in the analysis. The complete table is at the CDS.
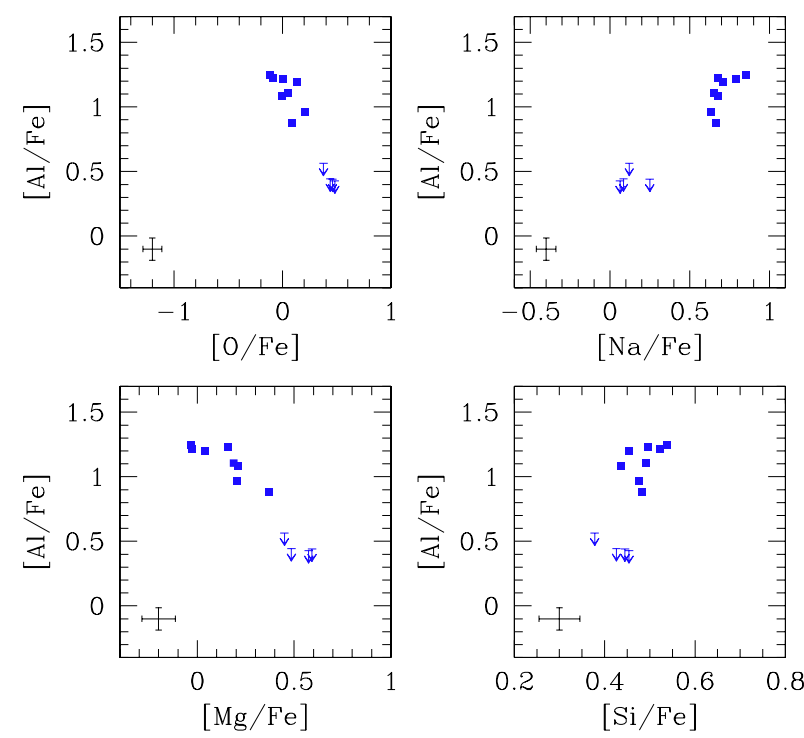

Fig. 10. Relations of $\mathrm{Al}$ with the proton-capture elements $\mathrm{O}, \mathrm{Na}, \mathrm{Mg}$, and Si in giants of NGC 4833 observed with UVES spectra. Error bars represent internal errors associated to the UVES sample. The upper limits in $\mathrm{Al}$ abundances are indicated by arrows.

position instead reveals clear patterns (Fig. 10), with the typical correlations and anticorrelations among elements produced or destroyed, respectively, by the interplay of the $\mathrm{Ne}-\mathrm{Na}$ and $\mathrm{Mg}-\mathrm{Al}$ cycles (Denisenkov \& Denisenkova 1989; Langer et al. 1993). Moreover, the sample, albeit limited, splits into two clearly separated groups.

The Mg-Si anticorrelation in Fig. 8, together with the Si-Al correlation (Fig. 10), already shows that some Si production occurred in the polluters of the first stellar generation in NGC 4833. To support this evidence further, we plot in Fig. 11 the $[\mathrm{Si} / \mathrm{Fe}]$ ratios as a function of the $\mathrm{O}$ and $\mathrm{Na}$ values. The evidence of a $\mathrm{Si}-\mathrm{O}$ anticorrelation is not robust for the GIRAFFE sample, but is clear for the more limited UVES sample. The correlation between $\mathrm{Si}$ and $\mathrm{Na}$ is represented in both samples well. The two
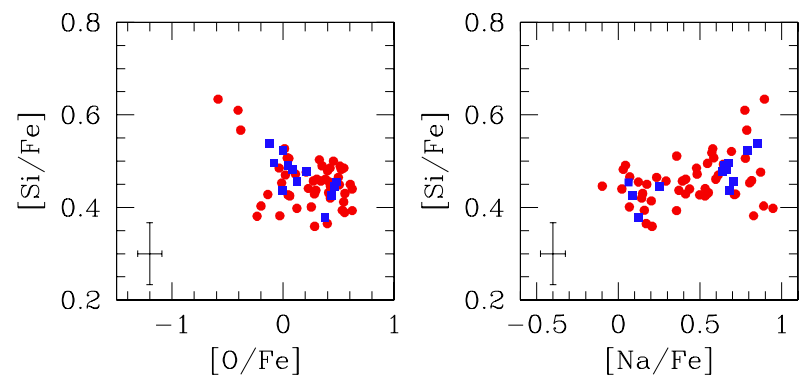

Fig. 11. Relations among proton-capture element ratios $[\mathrm{Si} / \mathrm{Fe}],[\mathrm{O} / \mathrm{Fe}]$, and $[\mathrm{Na} / \mathrm{Fe}]$ in NGC 4833 . Blue squares indicate stars of the UVES sample, red circles are for stars observed with GIRAFFE. The internal error bars refer to the latter sample.

stars with the lowest Mg abundances (Figs. 8 and 9) are also those showing the highest $\mathrm{Si}$ abundances, leaving no doubt that trends in $\mathrm{Si}$ and $\mathrm{Mg}$ are due to a common mechanism.

\subsection{Other elements}

The pattern of the $\alpha$-elements measured in NGC 4833 is shown as a function of the effective temperatures for individual stars in Fig. 12, including also species like $\mathrm{Mg}$ and $\mathrm{Si}$ involved in the proton-capture reactions discussed in the previous section.

The vertical scale, bracketing the range of $[\mathrm{Mg} / \mathrm{Fe}]$ ratios, is the same for all the elements to effectively show the large intrinsic dispersion of $\mathrm{Mg}$ and, partly, of $\mathrm{Si}$ with respect to other $\alpha$-elements with no intrinsic scatter in NGC 4833.

The run of elements of the Fe-peak $\mathrm{Sc}, \mathrm{V}, \mathrm{Cr}, \mathrm{Co}$, and $\mathrm{Ni}$ as a function of the temperature is shown in Fig. 13 for individual stars in NGC 4833 from the UVES and GIRAFFE samples. These elements present no surprise; they track iron, as in most GCs, with no trend as a function of the $T_{\text {eff }}$. The elements Mn and $\mathrm{Cu}$, only available for stars with UVES spectra, show the underabundance typical of metal-poor GCs (e.g. Simmerer et al. 2003; Sobeck et al. 2006). 

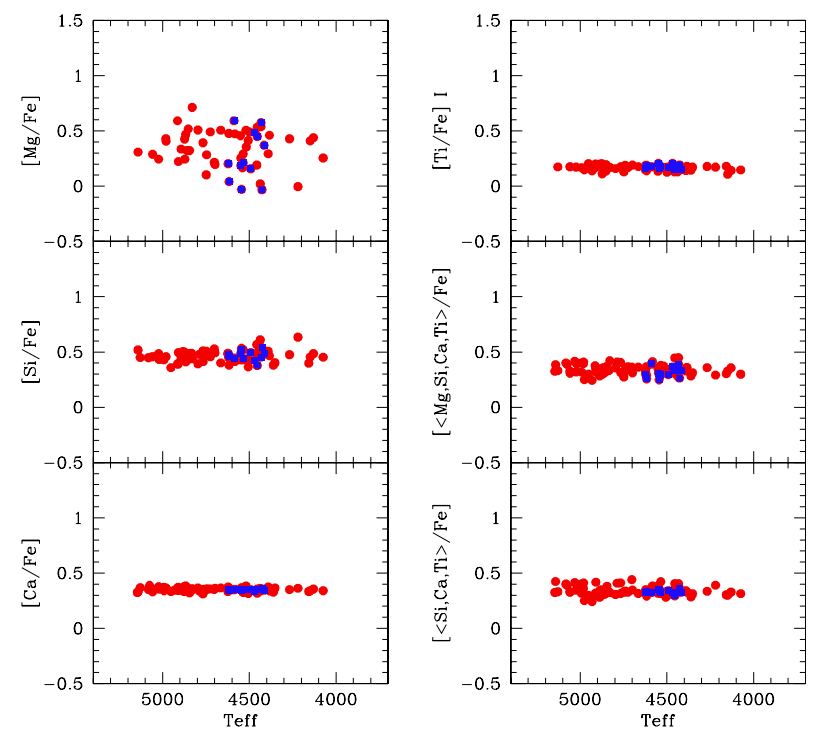

Fig. 12. Abundance ratios of $\alpha$-elements $\mathrm{Mg}, \mathrm{Si}, \mathrm{Ca}, \mathrm{Ti}$ I as a function of the effective temperature. The average of $[\alpha / \mathrm{Fe}]$ ratios are shown in the last two panels on the right column (including and excluding the $\mathrm{Mg}$ abundance from the mean, respectively). Blue squares are UVES stars. Internal error bars are provided in Tables 3 and 4.
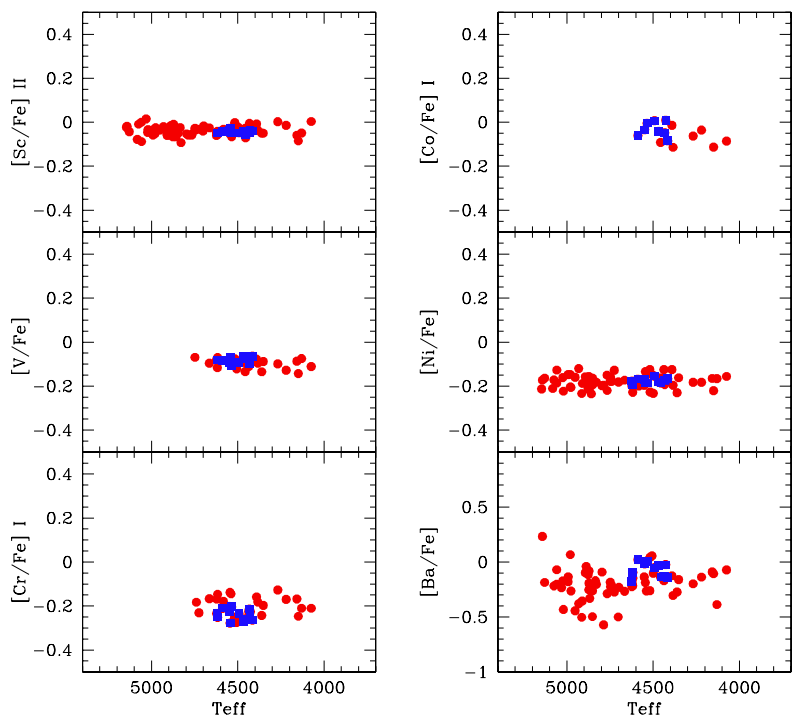

Fig. 13. Abundance ratios of elements of the Fe-peak ( $\mathrm{Sc}$ II, V, Cr, Co, $\mathrm{Ni}$ ) and of the neutron-capture element $\mathrm{Ba}$ as a function of the effective temperature. Blue squares are UVES stars. Note the different vertical scale for the panel with $\mathrm{Ba}$ abundances. Internal error bars are provided in Tables 3 and 4.

In the bottom right-hand panel of Fig. 13 we plot the abundance ratios of $\mathrm{Ba}$, the only neutron-capture element available for a large sample of stars in NGC 4833. As explained in Sect. 3.2, our finally adopted $\mathrm{Ba}$ abundances, displayed in this panel, are those obtained by using for all stars a fixed model metal abundance $([\mathrm{Fe} / \mathrm{H}]=-2.02$ dex, the average from the UVES spectra), and the microturbulence from the relation $v_{\mathrm{t}}=2.386-0.3067 \log g$ derived by Worley et al. (2013) for giants in the metal-poor GC M 15.

As recently shown in Worley et al. (2013) and Carretta et al. (2013a), this approach is quite effective in eliminating any trends of $\mathrm{Ba}$ abundances as a function of $v_{\mathrm{t}}$ and in reducing the ensuing spurious large scatters of the average. Only for Ba do we

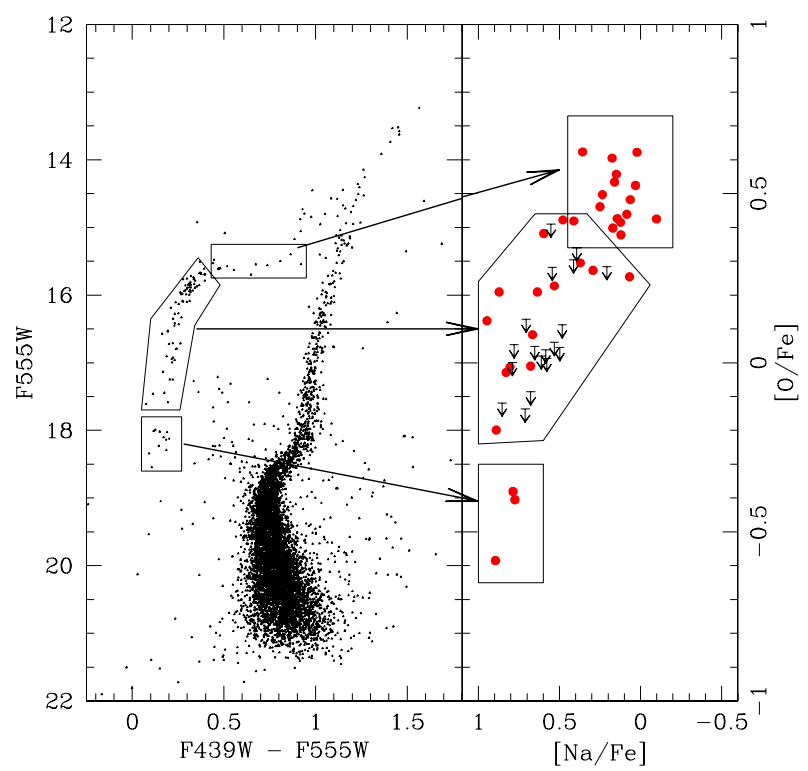

Fig. 14. HST colour-magnitude CMD of NGC 4833 from the snapshot survey by Piotto et al. (2002, left). The selected regions along the distribution of $\mathrm{HB}$ stars are tentatively associated to different groups along the $\mathrm{Na}-\mathrm{O}$ anticorrelation from the present work (right panel).

then adopt these values, since our intent is simply to state that this neutron-capture element from the $s$-process in NGC 4833 (i) does not have an intrinsic dispersion (compare the rms scatters of the means in Table 5 with the internal errors in Tables 3 and 4); and (ii) there is no relation between $\mathrm{Ba}$ and elements involved in proton-capture reactions.

We cannot estimate the relative contribution of the $r$ - and $s$-process of neutron capture from our data, because we did not measure a reliable abundance for the typical species that, like $\mathrm{Eu}$, primarily sample an almost pure $r$-process nucleosynthesis at all metal abundances. For the $[\mathrm{Ba} / \mathrm{Y}]$ ratio, we found an average value of 0.09 dex for NGC 4833 ( $\mathrm{rms}=0.06 \mathrm{dex}, 12$ stars), which is perfectly compatible with the ratios of field stars and GCs of similar metallicity (see e.g. Venn et al. 2004).

As for $\mathrm{Ba}$, we found no correlation or anti-correlation whatsoever between the abundances of the $s$-process element La and the abundances of proton-capture elements.

\section{Discussion}

The Na-O anticorrelation among RGB stars in NGC 4833 reaches a quite long extension. This result must not come unexpected. A statistically robust relation between the extent of the $\mathrm{Na}-\mathrm{O}$ anticorrelation and the hottest point along the $\mathrm{HB}$ is well known (Carretta et al. 2007c, 2010a). On the other hand, the HB in NGC 4833 presents a long blue tail, which stands out clearly, free of field contamination, in particular when high resolution HST imaging is used to construct the CMD (Fig. 14, left panel). Therefore, it would have been easy to predict a long Na-O anticorrelation in this cluster, which is exactly what we found with the current analysis.

It is tempting to associate the groups selected on the HB in the left-hand panel of Fig. 14 to the RGB stars distributed in the $\mathrm{Na}-\mathrm{O}$ anticorrelation (right panel of the same figure). After all, RGB stars should end up on the HB after igniting He burning at the centre. Qualitatively, the tentative correspondences illustrated in Fig. 14 may work well, provided that no strong radial gradients are present between our spectroscopic sample and the 
Table 10. Abundances of $n$-capture elements in stars of NGC 4833 with UVES spectra.

\begin{tabular}{lrcccccccc}
\hline \hline Star & $n$ & {$[\mathrm{Y} / \mathrm{Fe}] \mathrm{II}$} & $\mathrm{rms}$ & $n$ & {$[\mathrm{La} / \mathrm{Fe}] \mathrm{II}$} & $\mathrm{rms}$ & $n$ & {$[\mathrm{Nd} / \mathrm{Fe}] \mathrm{II}$} & $\mathrm{rms}$ \\
\hline 24081 & 10 & -0.15 & 0.11 & 4 & +0.30 & 0.16 & 4 & +0.45 & 0.09 \\
24252 & 6 & -0.24 & 0.07 & 5 & +0.35 & 0.12 & 4 & +0.47 & 0.05 \\
31163 & 10 & -0.11 & 0.08 & 3 & +0.25 & 0.07 & 4 & +0.36 & 0.10 \\
31332 & 10 & -0.09 & 0.10 & 4 & +0.40 & 0.13 & 4 & +0.39 & 0.11 \\
33347 & 9 & -0.19 & 0.09 & 3 & +0.30 & 0.04 & 4 & +0.44 & 0.10 \\
33554 & 10 & -0.13 & 0.09 & 4 & +0.25 & 0.15 & 4 & +0.42 & 0.09 \\
35680 & 5 & -0.09 & 0.06 & 3 & +0.35 & 0.06 & 4 & +0.40 & 0.11 \\
36402 & 7 & -0.24 & 0.09 & 2 & +0.25 & 0.22 & 3 & +0.44 & 0.04 \\
36454 & 9 & -0.16 & 0.13 & 3 & +0.25 & 0.11 & 3 & +0.36 & 0.07 \\
36484 & 9 & -0.12 & 0.09 & 3 & +0.40 & 0.06 & 4 & +0.41 & 0.13 \\
37197 & 9 & -0.23 & 0.14 & 2 & +0.50 & 0.05 & 3 & +0.40 & 0.15 \\
37498 & 9 & -0.10 & 0.10 & 4 & +0.25 & 0.07 & 4 & +0.46 & 0.07 \\
\hline
\end{tabular}

Notes. $n$ is the number of lines used in the analysis. The complete table is at the CDS.

Table 11. Abundances of Ba II in stars of NGC 4833.

\begin{tabular}{lccc}
\hline \hline Star & $N$ & {$[\mathrm{Ba} / \mathrm{Fe}] \mathrm{II}$} & $\mathrm{rms}$ \\
\hline 22810 & 1 & -0.10 & \\
23306 & 1 & -0.50 & \\
23437 & & & \\
23491 & 1 & -0.07 & \\
23518 & 1 & -0.33 & \\
24063 & 1 & -0.16 & \\
24252 & 3 & -0.13 & 0.01 \\
\hline
\end{tabular}

Notes. $n$ is the number of lines used in the analysis. The complete table is at the CDS.

photometric sample, which cover different parts of the cluster. More quantitative relations must, of course, await spectroscopic abundance analysis of in situ HB stars.

However, the major peculiarity we uncovered in NGC 4833 is maybe the wide spread in $\mathrm{Mg}$, whose abundance is clearly affected by large changes with respect to the usual plateau established by supernovae nucleosynthesis. As discussed in previous sections, we presented proofs that the Si abundance is partially modified by proton-capture reactions in this cluster also. This phenomenon was first observed by Yong et al. (2005) in NGC 6752, another metal-deficient GC with a long blue tail on the $\mathrm{HB}$, and afterwards individuated through $\mathrm{Si}-\mathrm{Al}$ correlations or Si-Mg anticorrelations in a number of other GCs (Carretta et al. 2009b). It is worth noting that in our large FLAMES survey, we found significant changes in the $\mathrm{Mg}$ (and $\mathrm{Si}$ ) abundances only in massive and/or metal-poor GCs.

The leakage from the $\mathrm{Mg}$ - $\mathrm{Al}$ cycle on ${ }^{28} \mathrm{Si}$ puts a strong constraint on the temperature at which H-burning occurred in the stars responsible for polluting the intracluster gas, because the reaction producing ${ }^{28} \mathrm{Si}$ becomes dominant when $T_{6} \sim 65 \mathrm{~K}$ (Arnould et al. 1999, where the temperature is expressed in millions of Kelvin).

As a result, although this constraint does not allow the type of stars providing the raw material for the formation of the second- generation to be distinguished (see Prantzos et al. 2007, their Fig. 8), a logical question is whether NGC 4833 is another case like NGC 2419, although scaled down in amount of the involved elemental variations. In the distant halo cluster NGC 2419, the third most massive GC in our Galaxy Cohen and Kirby (2012) and Mucciarelli et al. (2012) discovered a double population of stars on the RGB. One group is made of $\mathrm{Mg}$-normal giants with nearly solar abundance of potassium, whereas the other includes stars with a huge depletion of $\mathrm{Mg}$ (and large enhancement of $\mathrm{K}$ ) that apparently do not have a counterpart in any of the other Galactic GCs observed so far concerning $\mathrm{K}$ abundances (Carretta et al. 2013b).

In the scenario of multiple populations in GCs, Ventura et al. (2012) claim that the pattern of abundances observed in NGC 2419 may be explained also by proton-capture reactions occurring in a much higher temperature range than usually observed in more normal cluster stars, which is favoured by the low metallicity of the cluster. In these particular cases, the production of $\mathrm{Al}$ from the destruction of $\mathrm{Mg}$ would be accompanied by the activation of synthesis of heavier elements, such as K, $\mathrm{Ca}$, and also Sc by proton captures on Ar nuclei. The chief signature of this extreme burning would be the observation of anticorrelations between these elements and Mg, and in NGC 2419 we verified that the hypothesis by Ventura et al. agrees with observations (Carretta et al. 2013b).

NGC 4833 is far from being as massive as NGC 2419, however we observed a clear signature of processing in $\mathrm{H}$-burning at very high temperature in its abundance pattern; moreover, it is a metal-poor cluster. We then checked the run of $\mathrm{Si}, \mathrm{Sc}$, and $\mathrm{Ca}$ as a function of $\mathrm{Mg}$ abundances in NGC 4833, but no anti-correlation was found, apart from the one between $\mathrm{Si}$ and $\mathrm{Mg}$ already discussed above. There is only a hint of a correlation between $\mathrm{Ca}$ and Sc, which can be intriguing, because formally such a correlation may be expected if both these elements are produced by burning $\mathrm{Mg}$ under the conditions invoked by Ventura et al. (2012). Unfortunately, the associated internal errors are large with respect to the amount of the variations, and the correlation is scarcely statistically significant. (The Pearson correlation coefficient is only 0.25 ; with a number of degrees of freedom exceeding 70 this implies that the correlation is significant only at about $95-98 \%$ in two-tail tests.)

Therefore, NGC 4833 cannot be considered a true sibling of NGC 2419, which continues to represent a unicum among GCs. Nevertheless, we find in the present study that NGC 4833, with its associated extreme chemistry, stands out among other globular clusters. To illustrate this finding we used the $[\mathrm{Ca} / \mathrm{Mg}]$ vs. $[\mathrm{Ca} / \mathrm{H}]$ plane adopted in Carretta et al. (2013b) as a diagnostic for the relevance of the high temperature nuclear cycles possibly activated in polluters of the first generation in GCs. While measurements of the $\mathrm{K}$ abundance are still scarce, $\mathrm{Ca}$ and $\mathrm{Mg}$ are measured for a large number of stars, providing the precious advantage of large statistics.

In Fig. 15 we updated this diagnostic plot by adding stars from NGC 4833 analysed in the present paper. Typically, the $[\mathrm{Ca} / \mathrm{Mg}]$ ratios in $\mathrm{GC}$ stars show a narrow spread over a range of about 2 dex in $\mathrm{Ca}$, with a few exceptions, represented by a few giants in $\omega$ Cen and M 54. These are the two most massive clusters in the Galaxy, considered to be the nuclei left from dwarf galaxies accreted in the past. The three $\mathrm{Mg}$-poor stars in NGC 2808 (Carretta et al. 2009b) stand out around $[\mathrm{Ca} / \mathrm{H}] \sim-0.85$ dex, while in the low metallicity regime, the stars of NGC 4833 show a large dispersion in $[\mathrm{Ca} / \mathrm{Mg}$ ] when compared to other GCs. The spread in NGC 4833 does not reach the high values of the peculiar $\mathrm{Mg}$-poor component in NGC 2419; however, the stars in NGC 4833 with the largest depletion in $\mathrm{Mg}$ reach the same level of the most $\mathrm{Mg}$-poor stars in $\omega$ Cen. We caution the reader that among GCs stars, small offsets could exist with respect to the samples by Cohen \& Kirby, Mucciarelli et al., and Norris \& Da Costa, whereas all other RGB stars are from the homogeneous analysis by our group. There is no doubt that NGC 4833 shares some of the peculiarities also seen in $\omega$ Cen, M 54, and NGC 2808, although all these 


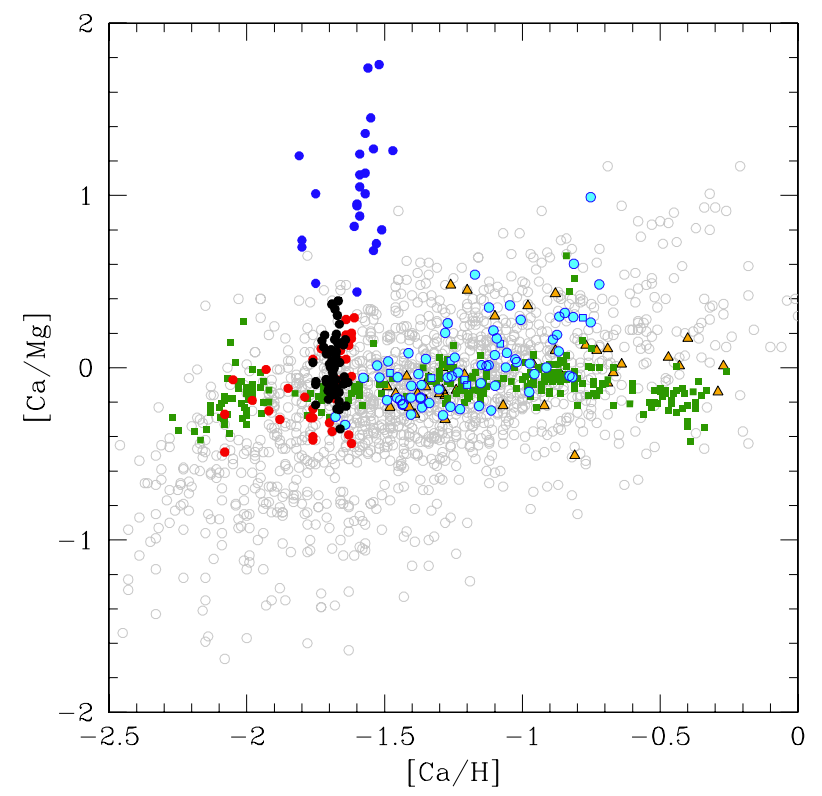

Fig. 15. $[\mathrm{Ca} / \mathrm{Mg}]$ ratio as a function of the $\mathrm{Ca}$ abundances for several stellar populations, adapted from Carretta et al. (2013b). Grey open circles are stars in eight dwarf spheroidal Milky Way satellite galaxies (Kirby et al. 2011); blue and red filled circles are RGB stars in NGC 2419 with $[\mathrm{Mg} / \mathrm{Fe}]$ lower and larger than 0.0 dex, respectively, from Cohen \& Kirby (2012) and Mucciarelli et al. (2012). Orange triangles are giants in $\omega$ Cen (Norris \& Da Costa 1995), cyan circles are RGB stars in M 54 (Carretta et al. 2010b). Green squares are for giants in 22 Galactic GCs (Carretta et al. 2009b, 2010c, 2011, 2013a,b). Black symbols are stars in NGC 4833 from the present study (squares and circles for the UVES and GIRAFFE samples, respectively).

GCs are far from reaching the huge $\mathrm{Mg}$-depletions observed in NGC 2419.

All these objects are high mass clusters, while the extension of the $\mathrm{HB}$ and of the Na-O anticorrelation (and, overall, of the proton-capture processing) are quite large in NGC 4833 with respect to its absolute magnitude. We could therefore wonder why NGC 4833 is not as massive.

In Fig. 7 we indicated the position of NGC 288 in the relation between the extension of the $\mathrm{Na}-\mathrm{O}$ anticorrelation and total cluster mass (luminosity). In Carretta et al. (2010a), we discussed the evidence that GCs lying left of this relation lost a larger-thanaverage fraction of their mass after their formation. This conclusion stemmed from old, classical proofs (such as tidal tails associated to NGC 288, Leon et al. 2000) or from a new interpretation of independent observations (e.g. the number density of X-ray sources in M 71, see Sect. 5.3 in Carretta et al. 2010a).

Is there any indication of a huge mass loss also in NGC 4833? The field of view around NGC 4833 is very crowded (see Fig. 1), with variable reddening, and this probably deterred investigations looking for tidal tails around GCs, because we are not aware of any studies of this kind for NGC 4833.

Another approach could be to look for clues from the present-day mass function (PDMF). Unfortunately, there seems to be no determination of the PDMF for NGC 4833, again probably bacause of the difficulties related to the above-mentioned conditions. We note that GCs with central brightness $\mu_{V}$, concentration, and central density $\rho_{0}$ similar to those of NGC 4833 present a PDMF with a rather steep slope of about -1 (de Marchi \& Pulone 2007; Paust et al. 2010), indicating many low mass stars, and therefore little evidence of mass loss.

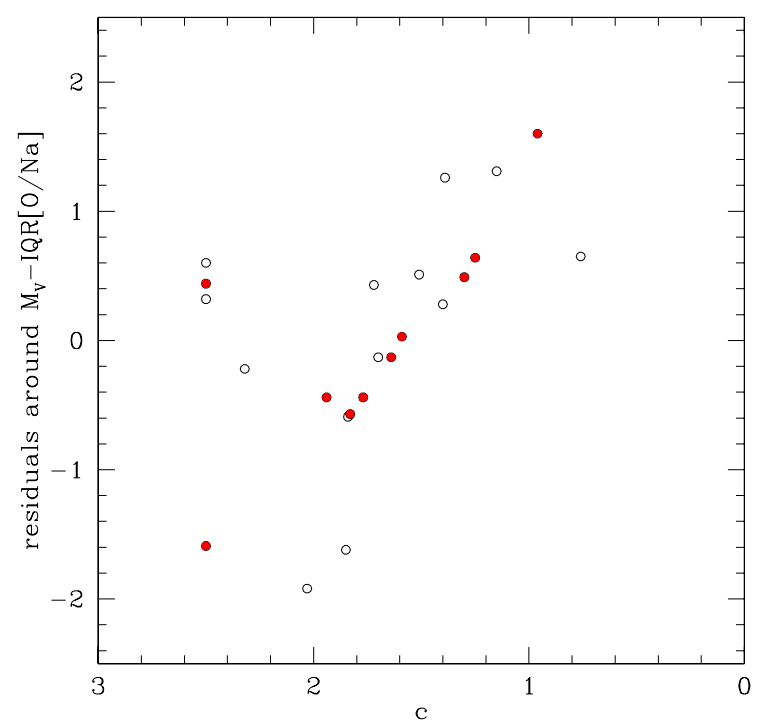

Fig. 16. Residuals around the relation between total absolute cluster magnitude $M_{V}$ and the interquartile range IQR[O/Na] as a function of the cluster concentration $c$ for GCs from our FLAMES survey. Filled points indicate inner halo clusters, as defined in Carretta et al. (2010a).

However, these other GCs are characterised by less critical orbits. Dynamical considerations suggest that NGC 4833 could actually be in a phase of destruction because of the tidal interaction with the bulge of the Galaxy. The concentration of NGC 4833 is modest ( $c=1.25$, Harris 1996), and it is worth noting that the cluster has a very eccentric orbit $(e \sim 0.84$; Casetti-Dinescu et al. 2007) passing very close to the Galactic bulge. This is probably a lethal combination for a cluster.

We inserted the data for NGC 4833 (from Harris 1996; Casetti-Dinescu et al. 2007) in Eq. (2) of Dinescu et al. (1999) that estimates the inverse ratio of the destruction time due to bulge shocking. For NGC 4833 we derived a destruction time between 1 and $3 \times 10^{8}$ years. The exact value depends on the mass of the Galactic bulge, assumed to be $34 \times 10^{9} M_{\odot}$ (Johnston et al. 1995), and on the velocity at the pericentre of the orbit, between 200 and $400 \mathrm{~km} \mathrm{~s}^{-1}$. The estimate may increase by a factor 2.5 by adopting a pericentre distance of $0.9 \mathrm{kpc}$, at the upper boundary of the range considered by Casetti-Dinescu et al. (2007), instead of $0.7 \mathrm{kpc}$. When compared to the other GCs included in the sample of Dinescu et al. (1999), NGC 4833 shows particularly critical parameters, making it a good candidate to strong tidal stripping and destruction by the bulge. Our estimate is approximate, but these findings are supported by Allen et al. (2006, 2008), who found that NGC 4833 has the sixth highest destruction rate among the 54 GCs analysed by them.

Incidentally, the low value of the $(M / L)_{V}$ we found (Sect. 2.1) also supports the hint of a significant loss of stars from the cluster, since energy equipartition should have favoured the loss of low mass stars.

The location of NGC 4833 and NGC 288 on the $M_{V}-I Q R$ diagram suggests that variations in cluster concentration $c$ can explain the observed scatter, at least in part. In Fig. 16 we plotted the residuals around the $M_{V}-I Q R$ correlation against the cluster concentration $c$. There is indeed a significant anti-correlation between these two quantities: the Pearson linear correlation coefficient is $r_{\mathrm{p}}=0.52$ that has a probability lower than $1 \%$ to be a random result. Of course, in this case it is better to use a bivariate analysis. The best fit is then

$I Q R=-0.16-(0.14 \pm 0.04) M_{V}-(0.16 \pm 0.08) c$. 
The rms of residuals around this regression is 0.18 , which compares well with typical internal errors in IQR $[\mathrm{O} / \mathrm{Na}]$. The correlation would further improve by excluding the core-collapse GCs, whose concentration is arbitrarily put at the constant value 2.5 in Harris (1996). The Pearson linear coefficient provided by this regression is $r_{\mathrm{p}}=0.65$. This result indicates that at a given absolute magnitude $M_{V}$, more loose clusters have a more extended $\mathrm{Na}-\mathrm{O}$ anticorrelation than more compact ones. This might seem to contradict the observation that second-generation stars are more centrally concentrated than first-generation ones, at least in several clusters (Gratton et al. 2012, and references therein). However, this may well be explained if the real parameter driving the $\mathrm{Na}-\mathrm{O}$ anticorrelation is the original rather than the present-day mass, and more loosely concentrated clusters have lost more mass than the more compact ones. The original mass of loosely concentrated clusters is therefore higher on average than for more compact clusters that now have the same $M_{V}$. More loose clusters are actually expected to be destroyed at a much faster rate by the tidal effects of the bulge and disk because the destruction rates for these mechanisms are expected to be proportional to the inverse of the cluster density (see Eqs. (1) and (2) in Dinescu et al. 1999).

If this argument is correct, IQR[O/Na] could be considered a better proxy for the original cluster mass than $M_{V}$. We might then possibly use the location of a cluster in the $M_{V}-I Q R$ diagram to infer not only its original mass, but also the amount of mass lost (assuming that very compact clusters only lose a small fraction of their original mass). This is quite a speculative but interesting possibility, which should be compared to other dynamical indicators of mass loss, such as the slope of the mass function. We intend to make such a comparison in a future study.

\section{Summary}

In the extension of our FLAMES survey of Na-O anticorrelation in GCs (e.g. Carretta et al. 2009a,b), we analysed FLAMES data for 78 RGB member stars of NGC 4833 (73 observed with GIRAFFE, 12 with UVES, and 7 in common). This is the first massive high-resolution spectroscopic study of this cluster. We confirmed that it is a metal-poor GCs, with an average $[\mathrm{Fe} / \mathrm{H}]=-2.04 \pm 0.003$ from the 73 GIRAFFE stars and $=-2.015 \pm 0.004$ from the 12 UVES stars. The rms dispersion is very small ( 0.024 and $0.014 \mathrm{dex}$, respectively), making NGC 4833 one of the most homogenous GC, at least in its general metallicity.

We obtained abundances of $\mathrm{Na}$ and $\mathrm{O}$ and concluded that the cluster presents the typical $\mathrm{Na}-\mathrm{O}$ anticorrelation found in (almost) all MW GCs (e.g. Carretta et al. 2010a; Gratton et al. 2012). The extension of the anticorrelation, as measured from the interquartile range of the $[\mathrm{O} / \mathrm{Na}]$ ratio, is large. This is in line with the expectations based on its very extended HB. The limited sample of UVES stars shows a marked bimodality in $\mathrm{Na}$ and $\mathrm{O}$ abundances, as well as in $\mathrm{Mg}, \mathrm{Al}, \mathrm{Si}$, with $\mathrm{P}$ and I,E stars clearly separated. The GIRAFFE sample only shows a hint of this separation, because of the larger errors in the abundances.

More exceptional is, however, the finding of large star-to-star variation in $\mathrm{Mg}$ abundance, anticorrelated with $\mathrm{Al}, \mathrm{Na}$, and $\mathrm{Si}$. The strong depletion in $\mathrm{Mg}$ implies nuclear processing at very high temperatures, exceeding a threshold equal to about 65 million Kelvin (Arnould et al. 1999). This has been found to date only in a few cases, mostly metal-poor and massive GCs, with the more extreme changes seen in objects such as the very massive $\omega$ Cen, M 54, and NGC 2419 (and to a lesser degree in NGC 2808). With a metallicity similar to NGC 4833, NGC 2419 is a more extreme case and clearly a unique case among GCs in the Milky Way. However, NGC 4833 reaches Mg depletion similar to the other massive clusters mentioned above, and it stands out among GCs of similar low metallicity.

This unusual chemical pattern, coupled with the position of NGC 4833 in the relation between IQR $[\mathrm{O} / \mathrm{Na}]$ and mass (total absolute magnitude), seems to indicate that the cluster was much more massive in the past. NGC 4833 has probably lost a conspicuous fraction of its original mass due to bulge shocking, as also indicated by its orbit.

Acknowledgements. We thank Chris Sneden for sending us the hyperfine structure components of many lanthanum lines, and we thank the referee for a careful reading of the manuscript and for suggestions. This publication makes use of data products from the Two Micron All Sky Survey, which is a joint project of the University of Massachusetts and the Infrared Processing and Analysis Center/California Institute of Technology, funded by the National Aeronautics and Space Administration and the National Science Foundation. This research has been funded by PRIN INAF 2011 "Multiple populations in globular clusters: their role in the Galaxy assembly" (PI E. Carretta), and PRIN MIUR 2010-2011, project "The Chemical and Dynamical Evolution of the Milky Way and Local Group Galaxies" (PI F. Matteucci). This research made use of the SIMBAD database, operated at the CDS, Strasbourg, France, and of NASA's Astrophysical Data System.

\section{References}

Allen, C., Moreno, E., \& Pichardo, B. 2006, ApJ, 652, 1150

Allen, C., Moreno, E., \& Pichardo, B. 2008, ApJ, 674, 237

Alonso, A., Arribas, S., \& Martinez-Roger, C. 1999, A\&AS, 140, 261

Alonso, A., Arribas, S., \& Martinez-Roger, C. 2001, A\&A, 376, 1039

Arnould, M., Goriely, S., \& Jorissen, A. 1999, A\&A, 347, 572

Bragaglia, A., Carretta, E., Gratton, R. G., et al. 2001, AJ, 121, 327

Bastian, N., Lamers, H. J. G. L. M., de Mink, S. E., et al. 2013a, MNRAS, 436, 2398

Bastian, N., Cabrera-Ziri, I., Davies, B., \& Larsen, S. S. 2013b, MNRAS, 436, 2852

Bellazzini, M., Bragaglia, A., Carretta, E., et al. 2012, A\&A, 538, A18

Cardelli, J. A., Clayton, G. C., \& Mathis, J. S. 1989, ApJ, 345, 245

Carretta, E. 2006, AJ, 131, 1766

Carretta, E., Bragaglia, A., Gratton R. G., et al. 2006, A\&A, 450, 523

Carretta, E., Bragaglia, A., Gratton, R.G., et al. 2007a, A\&A, 464, 967

Carretta, E., Bragaglia, A., Gratton, R. G., Lucatello, \& S. Momany, Y. 2007b, A\&A, 464, 927

Carretta, E., Recio-Blanco, A., Gratton, R.G., Piotto, G., Bragaglia, A. 2007c, ApJ, 671, L125

Carretta, E., Bragaglia, A., Gratton, R. G., et al. 2009a, A\&A, 505, 117

Carretta, E., Bragaglia, A., Gratton, R. G., \& Lucatello, S. 2009b, A\&A, 505, 139

Carretta, E., Bragaglia, A., Gratton, R. G., D’Orazi, V., \& Lucatello, S. 2009c, A\&A, 508, 695

Carretta, E., Bragaglia, A., Gratton, R. G., et al. 2010a, A\&A, 516, A55

Carretta, E., Bragaglia, A., Gratton, R. G., et al. 2010b, A\&A, 520, A95

Carretta, E., Bragaglia, A., Gratton, R., et al. 2010c, ApJ, 712, L21

Carretta, E., Lucatello, S., Gratton, R. G., Bragaglia, A., \& D’Orazi, V. 2011, A\&A, 533, A69

Carretta, E., Bragaglia, A., Gratton, R. G., Lucatello, S., \& D’Orazi, V. 2012a, ApJ, 750, L14

Carretta, E., Gratton, R. G., Bragaglia, A., D’Orazi, V., \& Lucatello, S. 2012b, A\&A, 550, A34

Carretta, E., Bragaglia, A., Gratton, R. G., et al. 2013a, A\&A, 557, A138

Carretta, E., Gratton, R. G., Bragaglia, A., et al. 2013b, ApJ, 769, 40

Casetti-Dinescu, D. I., Girard, T. M., Herrera, D., et al. 2007, AJ, 134, 195

Cohen, J. G., \& Kirby, E. N. 2012, ApJ, 760, 86

Cohen, J. G., \& Melendez, J. 2005, AJ, 129, 1607

D’Antona, F., Bellazzini, M., Caloi, V., et al. 2005, ApJ, 631, 868

Decressin, T., Meynet, G., Charbonnel, C., Prantzos, N., \& Ekstrom, S. 2007, A\&A, 464, 1029

Decressin, T., Baumgardt, H., \& Kroupa, P. 2008, A\&A, 492, 101

de Marchi, G., \& Pulone, L. 2007, A\&A, 467, 107

de Mink, S. E., Pols, O. R., Langer, N., \& Izzard, R. G. 2009, A\&A, 507, L1

Denisenkov, P. A., \& Denisenkova, S. N. 1989, A. Tsir., 1538, 11

D’Ercole, A., Vesperini, E., D’Antona, F., McMillan, S. L. W., \& Recchi, S. 2008, MNRAS, 391, 825

Dinescu, D. I., Girard, T. M., \& van Altena, W. F. 1999, AJ, 117, 1792 
D'Orazi, V., Biazzo, K., Desidera, S., et al. 2012, MNRAS, 423, 2789

D’Orazi, V., Campbell, S. W., Lugaro, M., et al. 2013, MNRAS, 433, 366

Figuera Jaimes, R., Arellano Ferro, A., Bramich, D. M., Giridhar, S., \& Kuppuswamy, K. 2013, A\&A, 556, A20

Garcia Lugo, G., Arellano Ferro, A., \& Rosenzweig, P. 2007, IAU Symp, 240, 214

Gratton, R. G. 1988, Rome Obs. Preprint Ser., 29

Gratton, R. G., \& Ortolani, S. 1989, A\&A, 211, 41

Gratton, R. G., Carretta, E., Eriksson, K., \& Gustafsson, B. 1999, A\&A, 350, 955

Gratton, R. G., Sneden, C., Carretta, E., \& Bragaglia, A. 2000, A\&A, 354, 169

Gratton, R. G., Carretta, E., Claudi, R., Lucatello, S., \& Barbieri, M. 2003, A\&A, 404, 187

Gratton, R. G., Sneden, C., \& Carretta, E. 2004, ARA\&A, 42, 385

Gratton, R. G., Lucatello, S., Bragaglia, A., et al. 2006, A\&A, 455, 271

Gratton, R. G., Lucatello, S., Bragaglia, A., et al. 2007, A\&A, 464, 953

Gratton, R. G., Carretta, E., \& Bragaglia, A. 2012, A\&ARv, 20, 50

Harris, W. E. 1996, AJ, 112, 1487

Johnson, C. I., \& Pilachowski, C. A. 2010, ApJ, 722, A1373

Johnson, C. I., \& Pilachowski, C. A. 2012, ApJ, 754, L38

Johnston, K. V., Spergel, D. N., \& Hernquist, L. 1995, ApJ, 451, 598

Kains, N., Bramich, D. M., Arellano Ferro, A., et al. 2013, A\&A, 555, A36

Kaluzny, J., Olech, A., Thompson, I., et al. 2000, A\&AS, 143, 215

Karakas, A. I., \& Lattanzio, J. C. 2003, PASA, 20, 279

King, I. R. 1966, AJ, 71, 64

Kraft, R. P., \& Ivans, I. I. 2003, PASP, 115, 143

Landolt, A. U. 1992, AJ, 104, 340

Langer, G. E., Hoffman, R., \& Sneden, C. 1993, PASP, 105, 301

Leon, S., Meylan, G., \& Combes, F. 2000, A\&A, 359, 907

Maccarone, T. J., \& Zurek, D. R. 2012, MNRAS, 423, 2

Mackey, A. D., \& van den Bergh, S. 2005, MNRAS, 360, 631

Magain, P. 1984, A\&A, 134, 189

Manfroid, J., Selman, F., \& Jones, H. 2001, The Messenger, 104, 16

Martin, N. F., Ibata, R. A., Chapman, S. C., Irwin, M. J., \& Lewis, G. F. 2007, MNRAS, 380, 281

Melbourne, J., \& Guhathakurta, P. 2004, AJ, 128, 271

Melbourne, J., Sarajedini, A., Layden, A., \& Martins, D. H. 2000, AJ, 120, 3127
Milone, A., Piotto, G., Bedin, L., et al. 2012, ApJ, 744, 58

Milone, A. P., Marino, A. F., Piotto, G., et al. 2013, ApJ, 767, 120

Minniti, D., Peterson, R. C., Geisler, D., \& Claria, J. J. 1996, ApJ, 470, 953

Momany, Y., Bedin, L. R., Cassisi, S., et al. 2004, A\&A, 420, 605

Mucciarelli, A., Bellazzini, M., Ibata, R., et al. 2012, MNRAS, 426, 2889

Murphy, B. W., \& Darragh, A. N. 2012, J. South Eastern Assoc. Res. Astron., 6, 72

Murphy, B. W., \& Darragh, A. N. 2013, AAS Meeting \#222,\#115.12

Norris, J. E., \& Da Costa, G. S. 1995, ApJ, 441, L81

Pasquini, L., Avila, G., Blecha, A., et al. 2002, The Messenger, 110, 1

Paust, N. E. Q., Reid, I. N., Piotto, G., et al. 2010, AJ, 139, 476

Piotto, G., King, I. R., Djorgovski, S. G., et al. 2002, A\&A, 391, 945

Piotto, G., Bedin, L., Anderson, J., et al. 2007, ApJ, 661, L53

Prantzos, N., Charbonnel, C., \& Iliadis, C. 2007, A\&A, 470, 179

Pryor, C., \& Meylan, G. 1993, ASP Conf. Ser., 50, 357

Ramirez, S., \& Cohen, J. G. 2002, AJ, 123, 3277

Ramirez, S., \& Cohen, J. G. 2003, AJ, 125, 224

Recio-Blanco, A., Aparicio, A., Piotto, G., De Angeli, F., \& Djorgovski, S. G. 2006, A\&A, 452, 875

Simmerer, J., Sneden, C., Ivans, I. I., et al. 2003, AJ, 125, 2018

Skrutskie, M. F., Skrutskie, M. F., Cutri, R. M., Stiening, R., et al. 2006, AJ, 131, 1163

Sneden, C., Cowan, J. J., Lawler, James, E., et al. 2003, ApJ, 591, 936

Sobeck, J. S., Ivans, I. I., Simmerer, J. A., et al. 2006, AJ, 131, 2949

Sollima, A., Bellazzini, M., \& Lee, J.-W. 2012, ApJ, 755, 156

Stetson, P. B. 1994, PASP, 106, 250

Valcarce, A. A. R., \& Catelan, M. 2011, A\&A, 533, A120

Valdes, F. G. 1998, Astronomical Data Analysis Software and Systems VII, eds. R. Albrecht, R. N. Hook, \& H. A. Bushouse, ASP Conf. Ser., 145, 53

Venn, K. A, Irwin, M., Shetrone, M. D., Tout, C. A., Hill, V., \& Tolstoy, E. 2004, AJ, 128, 1177

Ventura, P., D’Antona, F., Mazzitelli, I., \& Gratton, R. 2001, ApJ, 550, L65

Ventura, P., D’Antona, F., Di Criscienzo, M., et al. 2012, ApJ, 761, L30

Worley, C. C., Hill, V., Sobeck, J., \& Carretta, E. 2013, A\&A, 553, A47

Yong, D., Grundahl, F., Nissen, P. E., Jensen, H. R., \& Lambert, D. L. 2005, A\&A, 438, 875

Yong, D., Mélendez, J., Grundahl, F., et al. 2013, MNRAS, 434, 3452 